\title{
Rapid assessment of the ichthyofauna of the southern Guiana Shield tributaries of the Amazonas River in Pará, Brazil
}

\author{
Guilherme M. DUTRA ${ }^{1 *}$ (®) Tiago M. S. FREITAS ${ }^{2,3}$, Bruno S. PRUDENTE ${ }^{4}$, Gilberto N. SALVADOR ${ }^{3}$, \\ Manuela D. V. LEÃO ${ }^{5}$, Luiz A. W. PEIXOTO ${ }^{1}$, Marina B. MENDONÇA', André L. NETTO-FERREIRA ${ }^{7}$, \\ Fabio R. SILVA ${ }^{6}$, Luciano F. A. MONTAG ${ }^{3}$, Wolmar B. WOSIACKI ${ }^{6}$ \\ Museu de Zoologia da Universidade de São Paulo. Caixa Postal 42494. CEP 04218-970, São Paulo, SP, Brazil \\ Universidade Federal do Pará, Campus Universitário do Marajó, Faculdade de Ciências Naturais. CEP 68800-000, Breves, Pará, Brazil \\ Universidade Federal do Pará, Laboratório de Ecologia e e Conservação. Caixa Postal 479, CEP 66075-110, Belém, PA, Brazil \\ ${ }^{4}$ Universidade Federal Rural da Amazônia. CEP 68650-000, Capitão Poço, Pará, Brazil \\ Museu Nacional, Universidade Federal do Rio de Janeiro, Departamento de Vertebrados, Quinta da Boa Vista, CEP 20940-040, Rio de Janeiro, RJ, Brazil \\ ${ }^{6}$ Museu Paraense Emílio Goeldi, Campus de Pesquisa, Coordenação de Zoologia, Setor de Ictiologia. CEP 66077-830, Belém, Pará, Brazil \\ Universidade Federal do Rio Grande do Sul, Instituto de Biociências, Departamento de Zoologia, CEP 91501970, Porto Alegre, Rio Grande do Sul, Brazil \\ * Corresponding author. guilhermedutr@yahoo.com.br; $\mathbb{D}$ https://orcid.org/0000-0002-1010-3738
}

\section{ABSTRACT}

The Northern Pará Drainage System encompasses the left-bank tributaries of the Amazonas River in the southern Guiana Shield region of Pará state, Brazil. Five of the region's state protected areas are considered strategic to the conservation of its biodiversity. In the present study, we assessed the ichthyofauna of the five state protected areas of the Northern Pará Drainage System. Seven expeditions were conducted between January 2008 and January 2009, which surveyed stretches of the Cuminá, Cuminapanema, Curuá, Jari, Mapuera, Nhamundá, and Paru rivers. These surveys yielded 286 species belonging to 38 families and eight orders, including seven new records of fish species for Brazil, six of which are also new records for the Amazon basin. Our results provide a valuable database for future research and conservation programs in the protected areas of the region.

KEYWORDS: Northern Pará Drainage System, protected areas, Amazon, inventory, fish

\section{Avaliação rápida da ictiofauna em tributários do Rio Amazonas na porção sul do Escudo das Guianas no Pará, Brasil}

\section{RESUMO}

O Sistema de Drenagens do Norte do Pará abrange os afluentes da margem esquerda do Rio Amazonas na regiáo sul do Escudo das Guianas no estado do Pará, Brasil. Cinco das áreas estaduais protegidas da regiâo são consideradas estratégicas para a conservaçáo da biodiversidade. No presente estudo, nós acessamos a ictiofauna das cinco áreas de proteçáo estadual do Sistema de Drenagens do Norte do Pará. Sete expediçôes foram realizadas entre os meses de janeiro de 2008 e janeiro de 2009, amostrando trechos das bacias dos rios Cuminá, Cuminapanema, Curuá, Jari, Mapuera, Nhamundá e Paru. As expediçóes resultaram em 286 espécies pertencentes a 38 famílias e oito ordens, incluindo sete novos registros de peixes para o Brasil, seis deles também representando novos registros para a bacia Amazônica. Nossos resultados fornecem uma base sólida para futuros programas de pesquisa e conservação nas áreas de proteção da regiáo.

PALAVRAS-CHAVE: Calha Norte Paraense, áreas protegidas, Amazônia, inventário, peixes

\section{INTRODUCTION}

The Northern Pará Drainage System (NPDS) encompasses the left-bank tributaries of the Amazonas River in the southern Guiana Shield region of Pará state, Brazil. This area encompasses five strictly-protected or sustainable-use conservation units, as defined by the Brazilian National System of Conservation Units (Brasil 2000). Together with other types of federal protected areas, such as indigenous lands, the NPDS forms the Northern Pará Biodiversity Corridor, which connects the Central Amazonian Biodiversity Corridor with the Amapá

CITE AS: Dutra, G.M.; Freitas, T.M.S.; Prudente, B.S.; Salvador, G.N.; Leão, M.D.V.; Peixoto, L.A.W.; Mendonça, M.B.; Netto-Ferreira, A.L.; Silva, F.R.; Montag, L.F.A.; Wosiacki, W.B. 2020. Rapid assessment of the ichthyofauna of the southern Guiana Shield tributaries of the Amazonas River in Pará, Brazil. Acta Amazonica 50: 24-36. 
Biodiversity Corridor, forming the largest biodiversity corridor found anywhere in the world (SEMA 2011a). This corridor encompasses the world's largest complex of protected areas, with a total area of almost 22 million hectares, which corresponds to $78 \%$ of the total area of the NPDS (SEMA 2010).

The Northern Pará Drainage System includes a number of important left bank tributaries of the Amazonas, such as the Nhamundá, Trombetas, Paru, Cuminapanema, Cuminá, Curuá, and Jari rivers. These rivers drain from the southern border of the Guiana Shield - a region of relatively high altitudes and aquatic ecosystems with characteristic features, such as rapids and streams with rocky beds - to the lowlands of the lower Amazon basin. This altitudinal gradient and the environmental heterogeneity of the region have been interpreted as important biogeographical and ecological factors that contribute to the high biodiversity of Amazonian fish (Lujan et al. 2013; da Costa et al. 2018).

The ichthyofauna of the Guiana Shield was reviewed by Vari et al. (2009), who listed 1148 species. This geographic region comprises four freshwater ecoregions, delimited by Abell et al. (2008), known as the Orinoco Guiana Shield, Essequibo, the Guianas, and the Amazonas Guiana Shield. The latter ecoregion coincides with the southern portion of the Guiana Shield, and most of the NPDS. Vari et al. (2009) noted that the ichthyofauna of northern Pará is known only from the study of Ferreira (1993), who surveyed the Trombetas River basin intensively. Although the NPDS includes a number of other tributaries of the Amazonas River, its ichthyofauna is still poorly known, and has been referred to by Dagosta and de Pinna $(2017 ; 2019)$ as a under-sampled area that lacks adequate fish inventory data. Given the logistic difficulties of reaching many isolated parts of the Amazon basin, the rapid sampling approach has become an important strategy for the production of preliminary checklists from poorly-known, but potentially diverse ecosystems, such as those of the NPDS, providing essential data for the development of effective conservation policies and management plans (Rapp Py-Daniel et al. 2007; Bernard 2008).

Between 2008 and 2009, the Museu Paraense Emílio Goeldi conducted seven expeditions to the state protected areas located in the NPDS to inventory its fauna and flora. The results were used to develop the management plans of the protected areas (SEMA 2010; 2011a, b, c, d). Here, we present the checklist of the fish species sampled in the principal drainages of the five state protected areas of the Northern Pará Drainage System.

\section{MATERIAL AND METHODS}

\section{Sampled areas}

The present study focused on five state protected areas (Figure 1): Faro State Forest (FLOTA Faro), Trombetas State Forest (FLOTA Trombetas), Paru State Forest (FLOTA Paru), Grão-Pará Ecological Station (ESEC Grão-Pará), and
Maicuru Biological Reserve (REBIO Maicuru). The FLOTA Faro is the smallest of these conservation units, with an area of 635,936 ha, and is drained primarily by the Nhamundá River, which separates the Brazilian states of Amazonas and Pará (SEMA 2011b). The FLOTA Trombetas covers an area of $3,172,978 \mathrm{ha}$, and is drained, from west to east, by the Trombetas, Cuminá (also known as the Erepecuru or Paru do Oeste), and Cuminapanema rivers (SEMA 2011c). The ESEC Grão-Pará is the largest of the protected areas, with a total area of 4,245,819 ha, which is drained by the headwaters of the Mapuera River, and parts of the Trombetas, Cuminá, Cuminapanema, Curuá, and Maicuru basins (SEMA 2011a). The REBIO Maicuru has an area of 1,151,761 ha, and is drained partly by the Maicuru, Paru, and Jari river basins (SEMA 2011d), while the FLOTA Paru covers a total area of 3,612,914 ha, and is drained primarily by the Paru River basin (SEMA 2010).

With the exception of FLOTA Faro, which is located in the central Amazonian lowlands, the state protected areas of the NPDS are located in the southern Guiana Shield, at altitudes of 100 to $500 \mathrm{~m}$ above sea level, with areas of both Amazonian rainforest and savanna vegetation (Vari et al. 2009). The headwater streams and rivers of these foothills have clear water, with visibility of up to $5 \mathrm{~m}$, as observed in the Trombetas River. Most of the study area has a humid tropical climate (subtype Am in the Köppen classification), except for the northern extreme of ESEC Grão-Pará, which is classified as Aw (Peel et al. 2007).

\section{Fish sampling and species inventory}

We sampled 76 sites during seven expeditions to the five protected areas between January 2008 and January 2009 (Table 1; see Supplementary Material, Appendix S1 for information on each sampling site). The sampling sites included headwater streams, river channels and margins, and lakes (Figure 2). During the FLOTA Faro expedition, we sampled 11 sites in the Nhamundá River basin, while in the FLOTA Trombetas, we obtained samples from 13 sites in the Cuminá River basin. In REBIO Maicuru, we sampled 10 sites on the Ipitinga River, a tributary of the Jari River basin, while in FLOTA Paru, we sampled nine sites in the Paru River basin. Given the enormous size of ESEC Grão-Pará, three expeditions were conducted in this area, including 14 sites in the Curuá River basin, denominated here as the "ESEC G-P South" sector, 11 sites at the headwaters of the Mapuera River (ESEC G-P North sector), and eight sites in the Cuminapanema basin (ESEC G-P Center sector). The number of points sampled in each type of environment (streams, river channels and margins, and lakes) at each locality is shown in Table 1.

Fish specimens were collected using standard fishing gear (hand nets, seine nets, and gill nets). The hand nets were circular, approximately $60 \mathrm{~cm}$ in diameter, with a $3-\mathrm{mm}$ mesh. Seine nets of two sizes were used ( 2 or $5 \mathrm{~m}$ long), both $1.50 \mathrm{~m}$ high with a $5-\mathrm{mm}$ mesh. The gill nets varied in length 


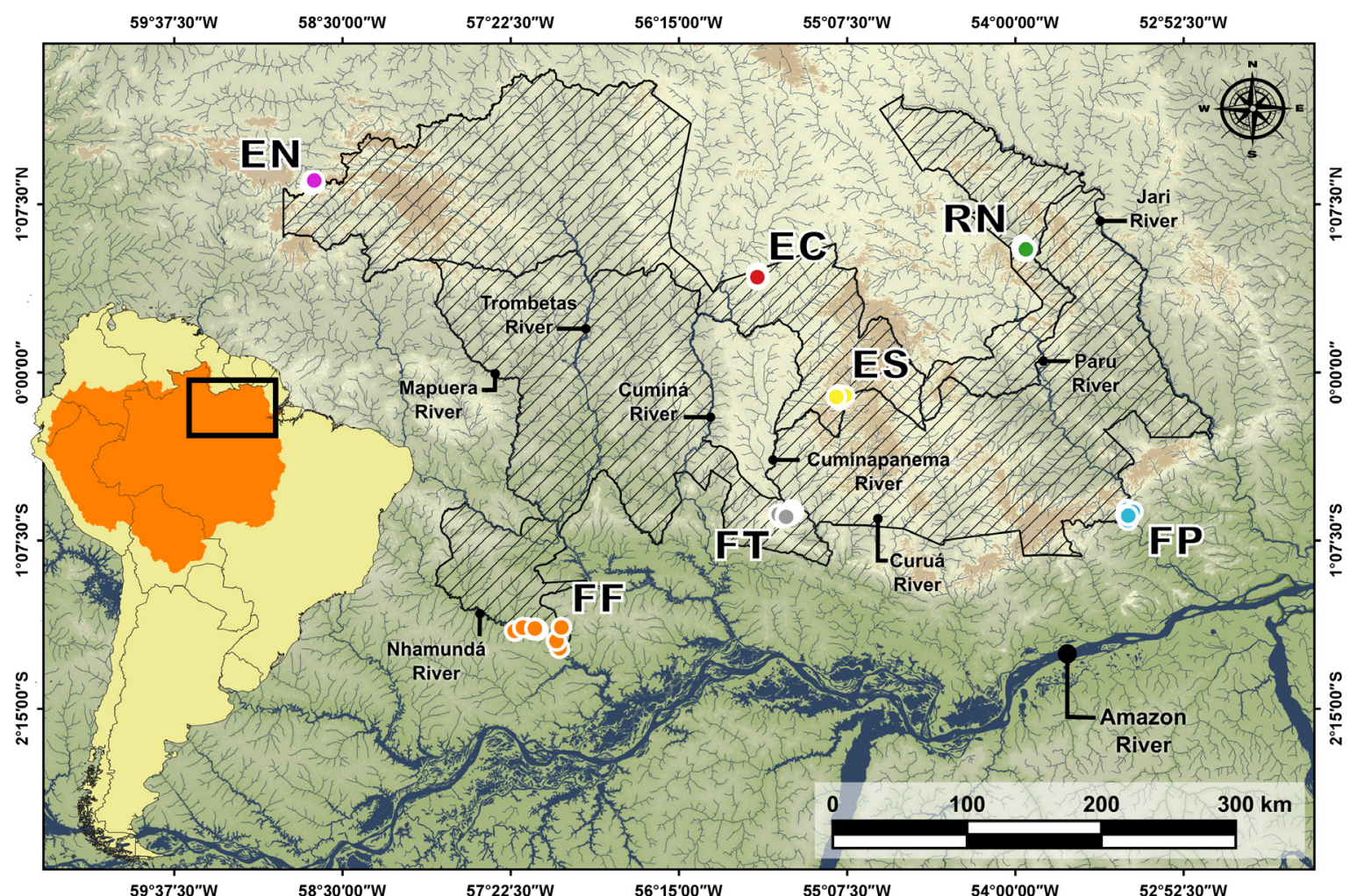

Figure 1. Map of the study area in the southern Guiana Shield, showing the tributaries of the Amazonas River in the Northern Pará Drainage System, in northern Brazil. The five state protected areas surveyed in the present study are hatched. The dots represent each of the sampling sites in the NPDS. FF = FLOTA Faro; EN = ESEC G-P North; EC = ESEC G-P Centre; FT = FLOTA Trombetas; ES = ESEC G-P South; RM = REBIO Maicuru; FP = FLOTA Paru. This figure is in color in the electronic version.

Table 1. Areas surveyed in the southern Guiana Shield tributaries of the Amazonas River in the five state protected areas of the Northern Pará Drainage System, in northern Brazil. Data on the elevation of each protected area were obtained from SEMA $(2010,2011$ a, b, c, d). m.a.s.l.= meters above sea level. The values in the "habitat type" column are the number of sampling points surveyed in each type of environment ( $\mathrm{St}=$ stream; $\mathrm{Lk}=$ lake; $\mathrm{Rc}=$ river channel; $\mathrm{Rm}=$ river $\mathrm{margin}$ ).

\begin{tabular}{|c|c|c|c|c|c|c|c|c|c|}
\hline \multirow{2}{*}{ Locality } & \multirow{2}{*}{ Month of survey } & \multirow{2}{*}{ Hydroperiod } & \multirow{2}{*}{ Drainage } & \multirow{2}{*}{ Elevation (m.a.s.l.) } & \multicolumn{4}{|c|}{ Habitat type } & \multirow{2}{*}{ Sampling methods } \\
\hline & & & & & St & Lk & Rc & $\mathrm{Rm}$ & \\
\hline FLOTA Faro & Jan 2008 & Rising water & Nhamundá River & $0-30$ & 2 & 5 & 4 & - & $\begin{array}{c}\text { hand net, seine and gill } \\
\text { nets }\end{array}$ \\
\hline FLOTA Trombetas & Apr 2008 & High water & Cuminapanema River & $300-450$ & 13 & - & - & 1 & hand net and seine \\
\hline ESEC G-P South & Jun 2008 & Ebbing water & Curuá River & $300-450$ & 9 & 1 & 4 & - & $\begin{array}{c}\text { hand net, seine and gill } \\
\text { nets }\end{array}$ \\
\hline ESEC G-P North & Sep 2008 & Low water & Mapuera River & $350-600$ & 11 & - & - & - & hand net and seine \\
\hline REBIO Maicuru & Oct 2008 & Low water & Ipitinga River & $150-250$ & 8 & - & - & 2 & $\begin{array}{c}\text { hand net, seine and gill } \\
\text { nets }\end{array}$ \\
\hline FLOTA Paru & Dec 2008 & Rising water & Paru River & $30-100$ & 6 & 1 & 2 & - & $\begin{array}{l}\text { hand net, seine and gill } \\
\text { nets }\end{array}$ \\
\hline ESEC G-P Centre & Jan 2009 & Rising water & Cuminá River & $300-450$ & 8 & - & - & - & hand net and seine \\
\hline
\end{tabular}

from 10 to $20 \mathrm{~m}$, with heights between 1.8 and $2.5 \mathrm{~m}$, and a range of mesh sizes $(20,40,50,60,70,80$ and $100 \mathrm{~mm})$.

Sampling effort was standardized among the different environments and collection methods. Hand and small $(2 \mathrm{~m})$ seine nets were used in streams, while the longer $(5 \mathrm{~m})$ seine nets were used in lakes and river margins, and the gill nets were set in lakes and rivers. At each stream, a stretch of $30 \mathrm{~m}$ was delimited, and four collectors each conducted 200 sweeps within the stretch, and the seine net was deployed 50 times. The lakes and river margins were also sampled with 50 sweeps of the seine net. The gill nets were set for approximately 24 hours, whenever possible, in lakes and river channels.

Once collected, the specimens were euthanized in a solution of clove oil, and fixed in $10 \%$ formalin for at 

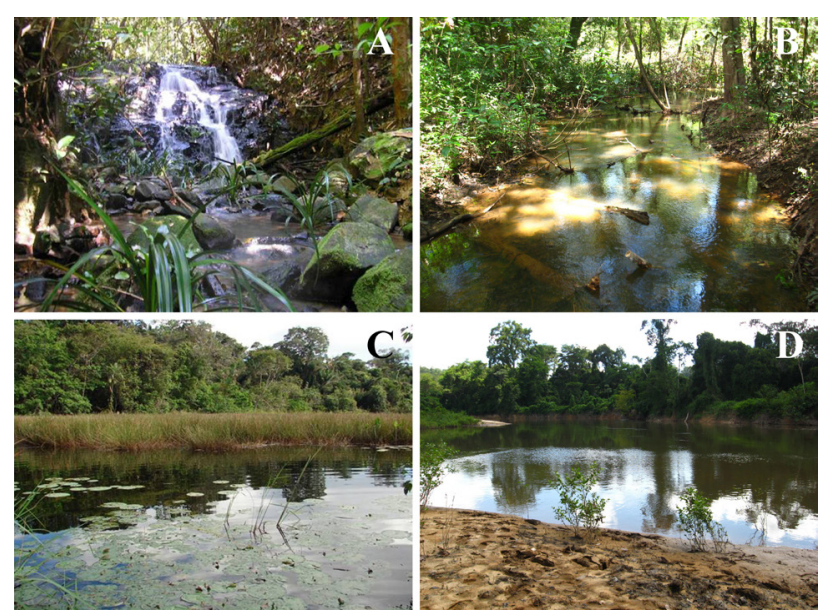

Figure 2. Examples of the habitats sampled in the RAP surveys conducted in the southern Guiana Shield tributaries of the Amazonas River in the five state protected areas of the Northern Pará Drainage System, in northern Brazil. (A) Rapids over rocky outcrops in a small stream in ESEC G-P North; (B) Forested stream in the REBIO Maicuru; (C) Lake in ESEC G-P South; (D) River margin in the middle stretch of the Ipitinga River in the REBIO Maicuru. This figure is in color in the electronic version.

least 48 hours before being transferred to $70 \%$ ethanol for storage. The specimens were identified to the lowest possible taxonomic level, using published identification keys, in the laboratory at the Museu Paraense Emílio Goeldi (MPEG) in Belém, northern Brazil, and the identifications were confirmed subsequently with the assistance of specialists. The nomenclature follows the current arrangement of Fricke et al. (2019). The species are listed alphabetically by family and organized systematically following Nelson et al. (2016). A complete list of the voucher specimens available in the fish collection of MPEG is also provided (Supplementary Material, Appendix S2). The extinction risk of each fish species was obtained from the list of threatened species of Pará state (Pará 2007), the Brazilian list of threatened species (Brasil 2014), and the IUCN Red List of Threatened Species (IUCN 2017). The species identified were also revised in the inventories of previous studies in the Amazonas tributaries of the Northern Pará Drainage System (Ferreira 1993; Vari et al. 2009; Dagosta and de Pinna 2019).

This research was authorized by the Brazilian System of Biodiversity Information and Authorization (SISBIO), through license number 4681-1. It was also approved by the Ethics Committee on the Use of Animals in Research of the Federal University of Pará, process CEUA 8293020418.

\section{RESULTS}

A total of 286 fish species were collected during the present study, representing 38 families and nine orders (Table 2; Figures 3 and 4). FLOTA Faro was the most diverse area, with 124 species in 31 families and eight orders. The vast majority of the species $(107,86.3 \%)$ recorded in FLOTA Faro were not recorded in any other area within the NPDS. The second most diverse area was FLOTA Paru, where 82 species were recorded ( 23 families, three orders). Once again, most of the species $(55,67.1 \%)$ recorded in FLOTA Paru were not registered at any of the other six sites. The surveys in REBIO Maicuru recorded 64 species (20 families, six orders), exactly half of which $(32,50 \%)$ were recorded only in this area of the NPDS. In turn, the surveys in FLOTA Trombetas recorded 28 species, representing 13 families and six orders. Nine of the species were restricted to this area, i.e., Corydoras sp., Pariolius armillatus Cope 1872, Ancistrus sp.3, Cteniloricaria napova Covain and Fisch-Muller 2012, Hemiancistrus sp., Paralithoxus sp., Peckoltia sp., Rineloricaria stewarti (Eigenmann 1909), and Anablepsoides sp.3. (Figures 5 and 6).

As already mentioned, ESEC Grão-Pará was surveyed during three expeditions. A total of 47 species were recorded in ESEC G-P South, representing 19 families and five orders. Eighteen of these species (corresponding to $38.3 \%$ of the fishes recorded in ESEC G-P South) were collected only in this sector. In ESEC G-P Center, 35 species were recorded, representing 16 families and five orders. Six of these species [Astyanax anterior Eigenmann 1908, Hyphessobrycon cf. agulha Fowler 1913, Jupiaba abramoides (Eigenmann 1909), Moenkhausia chrysargyrea (Günther 1864), Bryconops aff. affinis (Günther 1864), and Corydoras baderi Geisler, 1969] were collected only in this sector (Figure 4). Fourteen species were recorded in ESEC G-P North, representing nine families and five orders. Eight of these species [upiaba atypindi Zanata 1997, Bryconops cf. colaroja Chernoff and Machado-Allison 1999, Ancistrus sp.1, Ancistrus sp.2, Parotocinclus halbothi Lehmann, Lazzarotto and Reis 2014, Hypopomus artedi Kaup 1856, Anablepsoides sp.1 and "Aequidens" potaroensis Eigenmann 1912] were exclusive to this sector (Figures 5 and 6).

In addition to the species recorded in FLOTA Faro, which is located in the lowland Amazon basin, we recorded 179 species from the southern Guiana Shield tributaries of the Amazonas River. These species include 52 (29\% of the total) that were recorded in the region for the first time (see Table 2), of which seven had not previously been recorded in Brazil. The species recorded in Brazil for the first time were Hyphessobrycon georgettae Géry 1961, Jupiaba keithi (Géry, Planquette and Le Bail 1996), J. potaroensis (Eigenmann 1909), Phenacogaster simulata Lucena and Malabarba 2010, Corydoras guianensis Nijssen 1970, C. oxyrhynchus Nijssen and Isbrücker 1967, and Pariolius armillatus (Figures 5-6). With the exception of $P$. armillatus, all these species were also recorded in the Amazon basin for the first time. None of the fish species listed here for the NPDS are classified as endangered, either in the state of Pará (Pará 2007), in Brazil (Brasil 2014) or worldwide (IUCN 2017). 

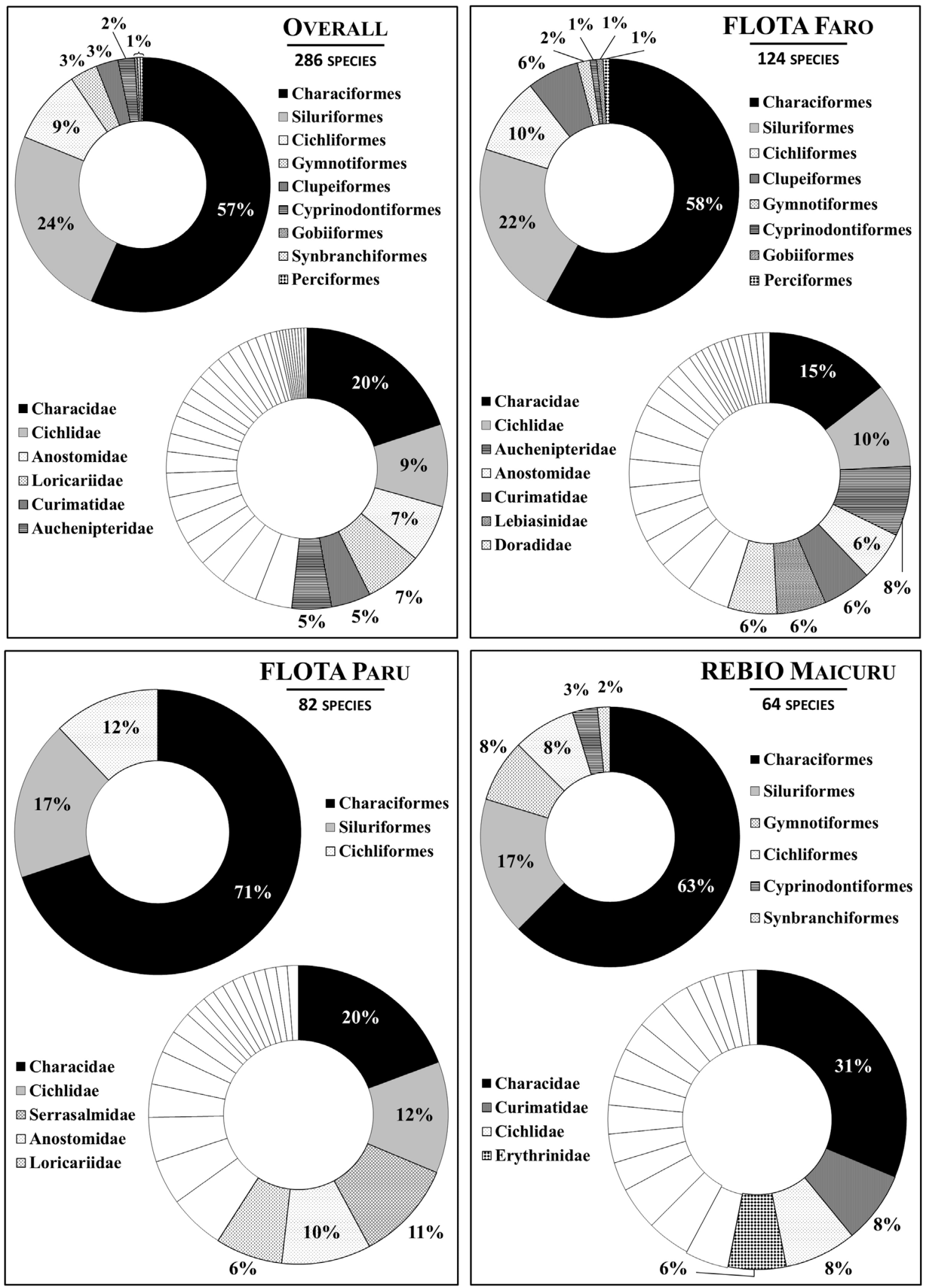

Figure 3. Percentage contribution of the different fish orders (upper graphic) and the most diverse families (lower graphic) to the inventory of the Northern Pará Drainage System (southern Guiana Shield tributaries of the Amazonas River) in the five state protected areas as a whole (Overall), and in FLOTA Faro, REBIO Maicuru, and FLOTA Paru. The blank segments represent families with less than $5 \%$ of the total species richness. 

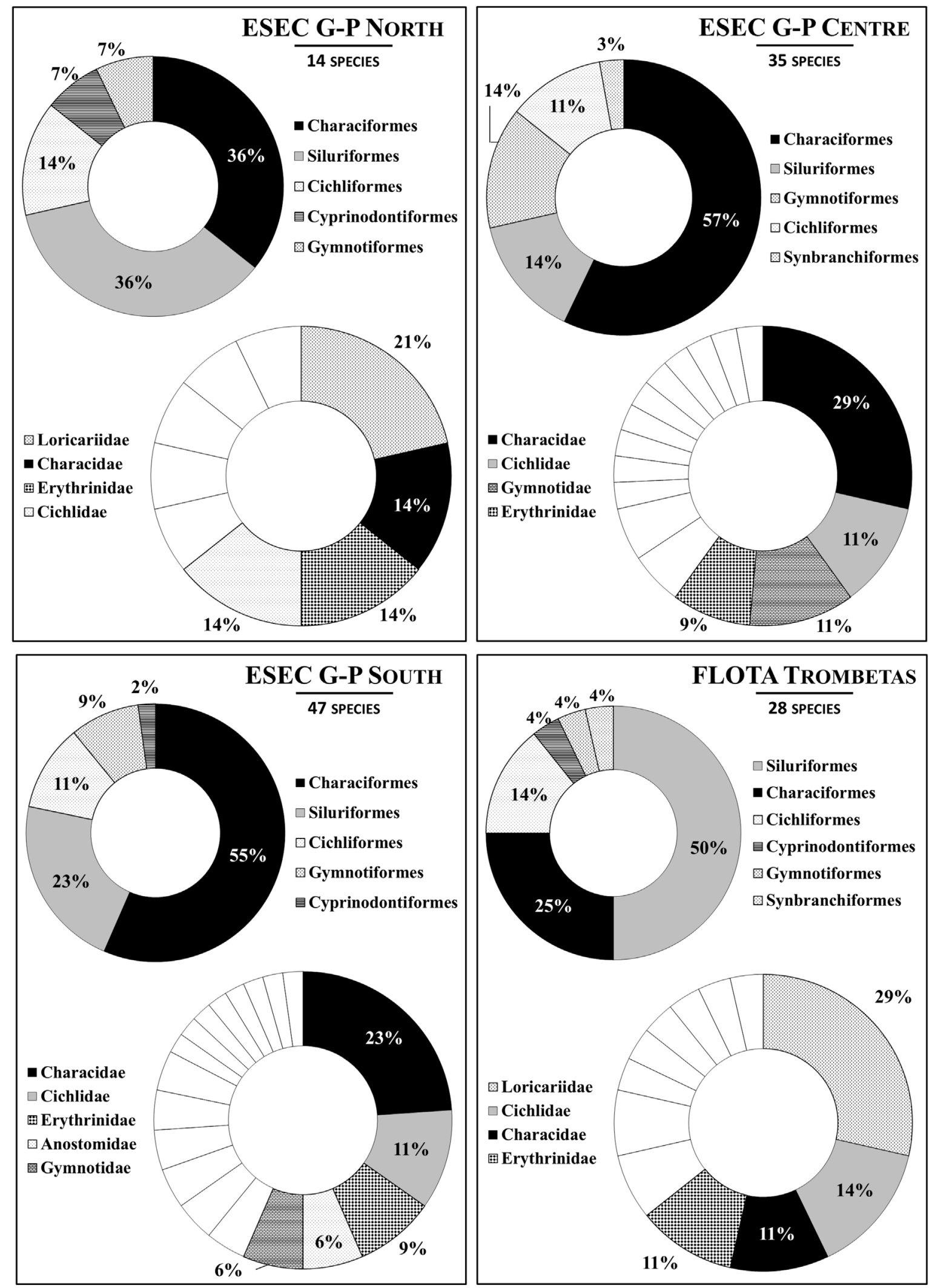

Figure 4. Percentage contribution of the different fish orders (upper graphic) and the most diverse families (lower graphic) to the inventories of ESEC Grão Pará and FLOTA Trombetas. The blank segments represent families with less than 5\% of the total species richness. 
Table 2. List of the fish species collected in the southern Guiana Shield tributaries of the Amazonas River in the five state protected areas (seven expeditions) of the Northern Pará Drainage System, in northern Brazil. FF = FLOTA Faro; FT = FLOTA Trombetas; ES = ESEC G-P South; EN = ESEC G-P North; RM = REBIO Maicuru; $F P=$ FLOTA Paru; EC $=$ ESEC G-P Center. ${ }^{*}=$ New record for the Northern Pará Drainage System.

\begin{tabular}{ll}
\hline Order/Family/Species & FF FT ES EN RM FP EC \\
\hline CLUPEIFORMES & \\
\hline Engraulidae & \\
\hline Amazonsprattus scintilla Roberts 1984 & X \\
Anchovia surinamensis (Bleeker 1865) & $\mathrm{X}$ \\
Anchoviella cayennensis (Puyo 1946) & $\mathrm{X}$ \\
Anchoviella guianensis (Eigenmann 1912) & $\mathrm{X}$ \\
Anchoviella lepidentostole (Fowler 1911) & $\mathrm{X}$ \\
\hline
\end{tabular}

\section{Pristigasteridae}

$\begin{array}{ll}\text { Ulisha amazonica (Miranda Ribeiro 1920) } & \text { X } \\ \text { Pellona castelnaeana Valenciennes 1847 } & \text { X } \\ \text { Pellona flavipinnis (Valenciennes 1837) } & \text { X }\end{array}$

\section{CHARACIFORMES}

\section{Acestrorhynchidae}

\begin{tabular}{lcc}
\hline Acestrorhynchus falcatus (Bloch 1794) & & X X X \\
Acestrorhynchus heterolepis (Cope 1878)* & & \\
Acestrorhynchus microlepis (Jardine 1841) & X & \\
Acestrorhynchus nasutus Eigenmann 1912 & X \\
Gnathocharax steindachneri Fowler 1913 & X & \\
Heterocharax macrolepis Eigenmann 1912 & X &
\end{tabular}

\section{Anostomidae}

Anostomoides laticeps (Eigenmann 1912)

Anostomus ternetzi Fernández-Yépez 1949 $x$

Hypomasticus lineomaculatus Birindelli, Peixoto,

Wosiacki and Britski 2013

Laemolyta proxima (Garman 1890)

Laemolyta taeniata (Kner 1858)

Leporinus agassizii Steindachner 1876

Leporinus amazonicus Santos and Zuanon 2008

Leporinus brunneus Myers1950

Leporinus cf. cylindriformis Borodin 1929

Leporinus fasciatus (Bloch 1794)

Leporinus friderici (Bloch 1794)

Leporinus cf. granti Eigenmann 1912

Leporinus jatuncochi Burns, Chatfield, Birindelli and

Sidlauskas 2017

Leporinus cf. megalepis (Günther 1863)

Leporinus melanostictus (Norman 1926)

Leporinus aff. nijsseni Garavello 1990

Leporinus tigrinus Borodin 1929*

Leporinus sp.1

Leporinus sp.2

Bryconidae

\begin{tabular}{lcc}
\hline Brycon pesu Müller and Troschel 1845 & $X$ & $X$ \\
\hline Chalceidae & \\
\hline Chalceus macrolepidotus Cuvier 1818* & $X$ \\
\hline
\end{tabular}

Table 2. Continued.

Order/Family/Species FF FT ES EN RM FP EC

\section{Characidae}

Acestrocephalus sardina (Fowler 1913)

Aphyocharax sp.

Astyanax anterior Eigenmann1908

Astyanax bimaculatus (Linnaeus 1758)*

Astyanax cf. multidens Eigenmann 1908

Brachychalcinus orbicularis (Valenciennes 1850)*

Brittanichthys myersi Géry 1965

Bryconamericus orinocoensis Román-Valencia 2003

Charax caudimaculatus Lucena 1987*

Hemibrycon surinamensis Géry $1962^{*}$

Hemigrammus analis Durbin 1909

Hemigrammus bellottii (Steindachner 1882)

Hemigrammus coeruleus Durbin 1908

Hemigrammus geisleri Zarske and Géry 2007*

Hemigrammus hyanuary Durbin 1918

Hemigrammus lunatus Durbin $1918^{*}$

Hemigrammus microstomus Durbin 1918

Hemigrammus ocellifer (Steindachner 1882)

Hemigrammus schmardae (Steindachner 1882)

Hemigrammus stictus (Durbin 1909)

Hemigrammus sp.1

Hemigrammus sp.2

Hyphessobrycon cf. agulha Fowler 1913

Hyphessobrycon diancistrus Weitzman 1977

Hyphessobrycon georgettae Géry 1961*

Hyphessobrycon socolofi Weitzman 1977

Jupiaba abramoides (Eigenmann 1909)*

Jupiaba acanthogaster (Eigenmann 1911)

Jupiaba atypindi Zanata 1997

1911)

Jupiaba keithi (Géry, Planquette and Le Bail 1996)*

Jupiaba meunieri (Géry, Planquette and Le Bail 1996)

Jupiaba polylepis (Günther 1864)

Jupiaba potaroensis (Eigenmann 1909)*

Knodus cf. heteresthes (Eigenmann 1908)

Knodus hypopterus (Fowler 1943)*

Knodus aff. hypopterus (Fowler 1943)

Knodus sp.1

Knodus sp.2

Microschemobrycon callops Böhlke 1953

Microschemobrycon casiquiare Böhlke 1953*

Moenkhausia celibela Marinho and Langeani 2010*

Moenkhausia chrysargyrea (Günther 1864)*

Moenkhausia collettii (Steindachner 1882)

Moenkhausia cotinho Eigenmann 1908

Moenkhausia megalops (Eigenmann 1907)

Moenkhausia mikia Marinho and Langeani 2010

Moenkhausia oligolepis (Günther 1864)

Moenkhausia aff. surinamensis Géry 1965

Phenacogaster simulata Lucena and Malabarba 2010*

Poptella brevispina Reis 1989

Rhinopetitia sp.

Roeboexodon guyanensis (Puyo 1948)*

Serrapinnus gracilis (Géry 1960)*

Tetragonopterus carvalhoi Melo, Benine, Mariguela and

Oliveira 2011

Tetragonopterus chalceus Spix and Agassiz 1829

Thayeria boehlkei Weitzman 1957*

$X$

$x$
$x$

$x^{x} \times x^{x}$

$x$

$x$

$x$
$x$

$X$
$X$

$x$

$x$

$x$
$x$
$x$

$x$

x

$x \quad x$

$\begin{array}{llll}x & & & \\ x & x & x \\ X & & & \\ x & & & \\ & x & & \\ x & & x & x \\ x & & x\end{array}$

$\begin{array}{ll}x & x \\ x & x\end{array}$

$x-x$

$x^{x}$

X

$x$

$x \quad x$

X

$x$
$x$

$x$

$x$

$x$
$x$

$x$

$x$

$x \quad x \quad x$

$x$

$x$

\begin{tabular}{|c|c|c|c|}
\hline & & & $x$ \\
\hline & & $x$ & X \\
\hline & & $x$ & $x \times$ \\
\hline & & & $x$ \\
\hline & & $x$ & X \\
\hline$x$ & $x$ & $x$ & $x$ \\
\hline & & $x$ & \\
\hline & & $x$ & \\
\hline & & $x$ & \\
\hline & & $x$ & \\
\hline & & & $x$ \\
\hline & & & \\
\hline
\end{tabular}


Table 2. Continued.

\begin{tabular}{|c|c|c|c|}
\hline \multirow{2}{*}{$\begin{array}{l}\text { Order/Family/Species } \\
\text { Chilodontidae }\end{array}$} & \multicolumn{3}{|c|}{ FF FT ES EN RM FP EC } \\
\hline & & & \\
\hline \multicolumn{2}{|l|}{ Caenotropus labyrinthicus (Kner1858) } & & $x$ \\
\hline \multicolumn{4}{|l|}{ Crenuchidae } \\
\hline Characidium cf. etheostoma Cope 1872 & $x$ & & $x$ \\
\hline Characidium zebra Eigenmann 1909 & $x$ & $x$ & $x$ \\
\hline Crenuchus spilurus Günther 1863 & $x$ & & \\
\hline Microcharacidium eleotrioides (Géry 1960)* & & $x$ & \\
\hline \multicolumn{4}{|l|}{ Ctenoluciidae } \\
\hline Boulengerella cuvieri (Spix and Agassiz 1829) & $x$ & & $x$ \\
\hline Boulengerella lucius (Cuvier 1816) & $x$ & & \\
\hline Boulengerella maculata (Valenciennes 1850) & $x$ & & \\
\hline Boulengerella xyrekes Vari 1995 & $x$ & & \\
\hline \multicolumn{4}{|l|}{ Curimatidae } \\
\hline Curimata knerii Steindachner 1876 & & & $x$ \\
\hline Curimata ocellata Eigenmann and Eigenmann 1889 & $x$ & & \\
\hline Curimatopsis macrolepis (Steindachner 1876) & $x$ & & \\
\hline $\begin{array}{l}\text { Curimatopsis melanura Dutra, Melo and Netto-Ferreira } \\
2018\end{array}$ & $x$ & & \\
\hline Cyphocharax abramoides (Kner 1858) & $x$ & & \\
\hline Cyphocharax aninha Wosiacki and Miranda 2013 & $x$ & $x$ & \\
\hline Cyphocharax festivus Vari 1992* & & & $x$ \\
\hline Cyphocharax helleri (Steindachner 1910) & & $x$ & \\
\hline Cyphocharax nigripinnis Vari 1992 & $x$ & & \\
\hline Cyphocharax cf. notatus (Steindachner 1908) & $x$ & & $x$ \\
\hline $\begin{array}{l}\text { Cyphocharax spiluropsis (Eigenmann and Eigenmann } \\
\text { 1889)* }\end{array}$ & $x \times$ & $x$ & $x \times$ \\
\hline Cyphocharax spilurus (Günther 1864) & $x$ & $x$ & $x$ \\
\hline Steindachnerina amazonica (Steindachner 1911)* & & $x$ & \\
\hline \multicolumn{4}{|l|}{ Cynodontidae } \\
\hline Cynodon septenarius Toledo-Piza 2000 & $x$ & & \\
\hline Hydrolycus armatus (Jardine 1841) & & & $x$ \\
\hline $\begin{array}{l}\text { Hydrolycus tatauaia Toledo-Piza, Menezes and Santos } \\
\text { 1999* }\end{array}$ & & & $x$ \\
\hline
\end{tabular}

\section{Erythrinidae}

Erythrinus erythrinus (Bloch and Schneider 1801) $\quad X \quad X \quad X \quad X \quad X \quad X$ Hoplerythrinus unitaeniatus (Spix and Agassiz 1829) $\quad X \quad X \quad X \quad X \quad X \quad X$ Hoplias aimara (Valenciennes 1847) $x$ Hoplias curupira Oyakawa and Mattox 2009* $\quad$ X X X $\quad X \quad X X$ Hoplias malabaricus (Bloch 1794) $\quad X \quad X \quad X \quad X \quad X$

\section{Gasteropelecidae}

\begin{tabular}{lll}
\hline Carnegiella marthae Myers 1927 & X & \\
Carnegiella strigata (Günther 1864) & X & \\
Gasteropelecus sternicla (Linnaeus 1758)* & & X \\
\hline
\end{tabular}

\section{Hemiodontidae}

\begin{tabular}{llc}
\hline Argonectes longiceps (Kner 1858) & X & \\
Bivibranchia fowleri (Steindachner 1908) & X & \\
Hemiodus argenteus Pellegrin 1909* & X & X \\
Hemiodus immaculatus Kner 1858 & X & \\
Hemiodus iratapuru Langeani and Moreira 2013 & & \\
Hemiodus semitaeniatus Kner 1858 & $X$ & $X$ \\
Hemiodus unimaculatus (Bloch 1794) & & \\
Micromischodus sugillatus Robert 1971 & X \\
\hline
\end{tabular}

Table 2. Continued.

Order/Family/Species FF FT ES EN RM FP EC

\section{Iguanodectidae}

Bryconops aff. affinis (Günther 1864)

Bryconops alburnoides Kner 1858

Bryconops aff. caudomaculatus (Günther 1864)

Bryconops aff. colanegra Chernoff and Machado-Allison

1999

Bryconops cf. colaroja Chernoff and Machado-Allison 1999

Bryconops cf. collettei Chernoff and Machado-Allison 2005

Bryconops disruptus Chernoff and Machado-Allison 1997 X

Bryconops giacopinii (Fernández-Yépez 1950)*

Bryconops aff. melanurus (Bloch 1794)

Bryconops sp.

Iguanodectes cf. geisleri Géry 1970

Iguanodectes cf. spilurus (Günther 1864)

\section{Lebiasinidae}

Copella arnoldi (Regan 1912)

Copella nattereri (Steindachner 1876)

Nannostomus digrammus (Fowler 1913)

Nannostomus eques Steindachner 1876

Nannostomus marginatus Eigenmann 1909

Nannostomus trifasciatus Steindachner 1876

Nannostomus unifasciatus Steindachner 1876

Pyrrhulina cf. maxima Eigenmann and Eigenmann 1889

Pyrrhulina sp.

\section{Parodontidae}

\section{Parodon guyanensis Géry 1960}

Prochilodontidae

Prochilodus rubrotaeniatus Jardine 1841

$x$

$x$

$x$

$x$

\section{Serrasalmidae}

Mylesinus paraschomburgkii Jégu, Santos and Ferreira $1989 \quad$ X

Myloplus arnoldi (Ahl 1936)*

Myloplus rhomboidalis (Cuvier 1818)

Myloplus rubripinnis (Müller and Troschel 1844)

Piaractus brachypomus (Cuvier 1818)*

Pygopristis denticulata (Cuvier 1819)

Serrasalmus eigenmanni Norman 1929

Serrasalmus hastatus Fink \& Machado-Allison, 2001*

Serrasalmus manueli (Fernández-Yépez and Ramírez 1967) X

Serrasalmus rhombeus (Linnaeus 1766)

$x$

$x \quad x$

$x$

$x$

$x$

$x$

\section{$x$}

$x$

$x$

Serrasalmus sp.

\section{Triportheidae}

Agoniates halecinus Müller and Troschel 1845

Triportheus albus Cope 1872

\section{SILURIFORMES}

\section{Auchenipteridae}

Ageneiosus inermis (Linnaeus 1766)*

Ageneiosus lineatus Ribeiro, Rapp Py-Daniel and Walsh 2017 X

Ageneiosus polystictus Steindachner 1915

Auchenipterichthys longimanus (Günther 1864)

Auchenipterus brachyurus Cope 1878

Auchenipterus nuchalis (Spix and Agassiz 1829)

Centromochlus heckelii (De Filippi 1853)

Tatia intermedia (Steindachner 1877)

$\begin{array}{llll}x & x \\ x & & & \\ X & & & \\ X & & & \\ x & & & \\ x & x & x & x \\ x & & & \end{array}$

$x$

Tatia nigra Sarmento-Soares and Martins-Pinheiro $2008 \times$

Tatia orca (Sarmento-Soares, Lazzarotto, Py-Daniel and $x$ Leitão 2017)

Trachelyichthys decaradiatus Mees 1974

Trachelyopterichthys taeniatus (Kner 1858)

Trachelyopterus galeatus (Linnaeus 1766)

\begin{tabular}{ll} 
& \\
$x$ & \\
$x$ & \\
$x$ & \\
$x$ & \\
$x$ & \\
$x$ & \\
$x$ & \\
$x$ & \\
$x$ & \\
$x$ & \\
$x$ & \\
& $x$ \\
\hline
\end{tabular}


Table 2. Continued.

\begin{tabular}{|c|c|c|c|c|c|c|}
\hline \multirow{2}{*}{$\begin{array}{l}\text { Order/Family/Species } \\
\text { Callichthyidae }\end{array}$} & \multicolumn{6}{|c|}{ FF FT ES EN RM FP EC } \\
\hline & & & & & & \\
\hline Callichthys callichthys (Linnaeus 1758) & & $x \times$ & $x$ & x & $x$ & \\
\hline Corydoras baderi Geisler 1969 & & & & & & $x$ \\
\hline Corydoras guianensis Nijssen 1970* & & & & $x$ & & \\
\hline Corydoras oxyrhynchus Nijssen and Isbrücker 1967* & & & & $x$ & & \\
\hline Corydoras sp. & x & & & & & \\
\hline Hoplosternum littorale (Hancock 1828) & $x$ & & & & & \\
\hline Megalechis picta (Müller and Troschel 1848) & $x$ & & & & & \\
\hline \multicolumn{7}{|l|}{ Cetopsidae } \\
\hline Helogenes marmoratus Günther 1863 & $x$ & $x \times$ & & $x$ & & $x$ \\
\hline
\end{tabular}

\begin{tabular}{|c|c|c|}
\hline Acanthodoras cataphractus (Linnaeus 1758) & $x$ & \\
\hline $\begin{array}{l}\text { Acanthodoras spinosissimus (Eigenmann and } \\
\text { Eigenmann 1888) }\end{array}$ & $x$ & \\
\hline Anduzedoras oxyrhynchus (Valenciennes 1821) & $x$ & \\
\hline Nemadoras elongatus (Boulenger 1898) & $x$ & \\
\hline Oxidoras niger (Valenciennes 1821)* & & $x$ \\
\hline Scorpiodoras heckelii (Kner 1855) & $x$ & \\
\hline Tenellus leporhinus (Eigenmann 1912) & $x$ & \\
\hline Trachydoras brevis (Kner 1853) & $x$ & \\
\hline
\end{tabular}

\section{Heptapteridae}

Gladioglanis conquistador Lundberg, Bornbusch and $x$

Mago-Leccia 1991

Imparfinis hasemani Steindachner 1915

Pariolius armillatus Cope $1872^{*}$

Pimelodella cristata (Müller and Troschel 1849)

Pimelodella geryi Hoedeman 1961

Pimelodella humeralis Slobodian, Akama and Dutra 2017

Pimelodella leptosoma (Fowler 1914)

Rhamdia quelen (Quoy and Gaimard 1824)* $\quad$ X X $\quad$ X

\section{Loricariidae}

Ancistrus sp.

Ancistrus sp.2

Ancistrus sp.3

Ancistrus sp.4

Baryancistrus hadrostomus Oliveira, Py-Daniel and

Oyakawa 2019

Cteniloricaria napova Covain and Fisch-Muller 2012

Curculionichthys sp.

Hemiancistrus sp.

Hypostomus carinatus (Steindachner 1881)

Hypostomus hemicochliodon Armbruster 2003*

Hypostomus sp.

Otocinclus vittatus Regan 1904*

$x$

$x$

$x$

Paralithoxus sp.

Parotocinclus halbothi Lehmann, Lazzarotto and Reis 2014

Peckoltia sp

Pseudancistrus sp.1

Pseudancistrus sp.2

Rineloricaria stewarti (Eigenmann 1909)*

Rineloricaria sp.

\section{Pimelodidae}

Hemisorubim platyrhynchos (Valenciennes 1840)

Hypophthalmus fimbriatus Kner 1858

Hypophthalmus marginatus Valenciennes 1840

Pimelodus albofasciatus Mees 1974

Pimelodus ornatus Kner 1858

Pinirampus pirinampu (Spix and Agassiz 1829)

Pseudoplatystoma punctifer (Castelnau 1855)

Sorubim elongatus Littmann, Burr, Schmidt and Isern 2001* X
Table 2. Continued.

Order/Family/Species FF FT ES EN RM FP EC

\section{Pseudopimelodidae}

Batrochoglanis villosus (Eigenmann 1912) X

Microglanis poecilus Eigenmann $1912 \quad X$

Trichomycteridae

Ituglanis amazonicus (Steindachner 1882)* $\quad$ X $\quad$ X $\quad X \quad X \quad X$

Paracanthopoma parva Giltay 1935

Stegophilus panzeri (Ahl 1931)*

$x$

Stenolicmus ix Wosiacki, Coutinho and Montag $2011 \quad$ X

\section{GYMNOTIFORMES}

\section{Gymnotidae}

Electrophorus voltai de Santana, Wosiacki, Crampton,

Sabaj, Dillman, Castro e Castro, Bastos and Vari 2019

Gymnotus carapo Linnaeus 1758

Gymnotus coropinae Hoedeman 1962

Gymnotus cf. stenoleucus Mago-Leccia 1994

Gymnotus sp.

$x$

\section{Hypopomidae}

Brachyhypopomus beebei (Schultz 1944)

Brachyhypopomus pinnicaudatus (Hopkins, Comfort,

Bastian and Bass 1990)*

Brachyhypopomus sullivani Crampton, de Santana,

Waddell and Lovejoy 2017

Hypopomus artedi (Kaup 1856)*

X

Rhamphichthyidae

Hypopygus lepturus Hoedeman 1962

\section{CYPRINODONTIFORMES}

\section{Rivulidae}

Anablepsoides sp.1

Anablepsoides sp.2

Anablepsoides sp.3

Laemosemion sp.1

Laemosemion sp.2

Laemosemion sp. 3

\section{GOBIIFORMES}

\section{Eleotridae}

Microphilypnus ternetzi Myers 1927

\section{SYNBRANCHIFORMES}

\section{Synbranchidae}

\begin{tabular}{llll}
\hline Synbranchus marmoratus Bloch 1795 & X & X & X \\
\hline
\end{tabular}

\section{CICHLIFORMES}

\section{Cichlidae}

Acarichthys heckelii (Müller and Troschel 1849)

Acaronia nassa (Heckel 1840)

"Aequidens" potaroensis Eigenmann 1912*

Aequidens tetramerus (Heckel 1840)

Aequidens sp. 1

Aequidens sp.2

Aequidens sp.3

Apistogramma agassizii (Steindachner 1875)

Apistogramma cf. pertensis (Haseman 1911)

Apistogramma gr. regani Kullander 1980

Apistogramma sp.

Caquetaia spectabilis (Steindachner 1875)

Cichla jariina Kullander and Ferreira 2006

Crenicichla albopunctata Pellegrin 1904*

Crenicichla cf. inpa Ploeg 1991

Crenicichla menezesi Ploeg 1991*

Crenicichla pydanielae Ploeg 1991

\begin{tabular}{ccc} 
& $x$ & \\
$x$ & $x$ & $x$ \\
& $x$ & $x$ \\
$x$ & & $x$ \\
$x$ & & $x$ \\
\hline
\end{tabular}

$x$

$x$

$x \quad x$

$x^{x^{x}}$

$x$

X

$x$

$x$

\begin{tabular}{|c|c|c|c|}
\hline$X$ & & & \\
\hline \multicolumn{4}{|l|}{$X$} \\
\hline \multicolumn{4}{|c|}{$X$} \\
\hline \multicolumn{4}{|l|}{$X$} \\
\hline & $X$ & $X$ & $X$ \\
\hline & $X$ & & \\
\hline$X$ & & & $X X$ \\
\hline
\end{tabular}

\begin{tabular}{|c|c|c|c|}
\hline$X$ & & & \\
\hline$x$ & & & \\
\hline \multicolumn{4}{|l|}{$X$} \\
\hline & & $X$ & \\
\hline \multirow[t]{3}{*}{$X$} & & $x$ & \\
\hline & & $x$ & \\
\hline & $x$ & & \\
\hline$x \times$ & $x$ & $X$ & $x$ \\
\hline & & & $x$ \\
\hline & & & $X$ \\
\hline
\end{tabular}


Table 2. Continued

\begin{tabular}{|c|c|c|c|c|}
\hline Order/Family/Species & FF FT & EN & RM & FP EC \\
\hline Crenicichla regani Ploeg 1989 & $X$ & & & \\
\hline Crenicichla saxatilis (Linnaeus 1758)* & $x$ & $x$ & $x$ & $x$ \\
\hline Crenicichla strigata Günther 1862 & & & & $x$ \\
\hline Geophagus altifrons Heckel 1840 & & & & $x$ \\
\hline Krobia guianensis (Regan 1905)* & & & $x$ & \\
\hline Mesonauta acora (Castelnau 1855) & $x$ & & & \\
\hline Mesonauta festivus (Heckel 1840) & $x$ & & & $x$ \\
\hline Satanoperca acuticeps (Heckel 1840) & X & & & \\
\hline Satanoperca jurupari (Heckel 1840) & $x$ & & & $x$ \\
\hline Taeniacara candidi Myers 1935 & $x$ & & & \\
\hline \multicolumn{5}{|l|}{ PERCIFORMES } \\
\hline \multicolumn{5}{|l|}{ Sciaenidae } \\
\hline Plagioscion squamosissimus (Heckel 1840) & $x$ & & & \\
\hline
\end{tabular}

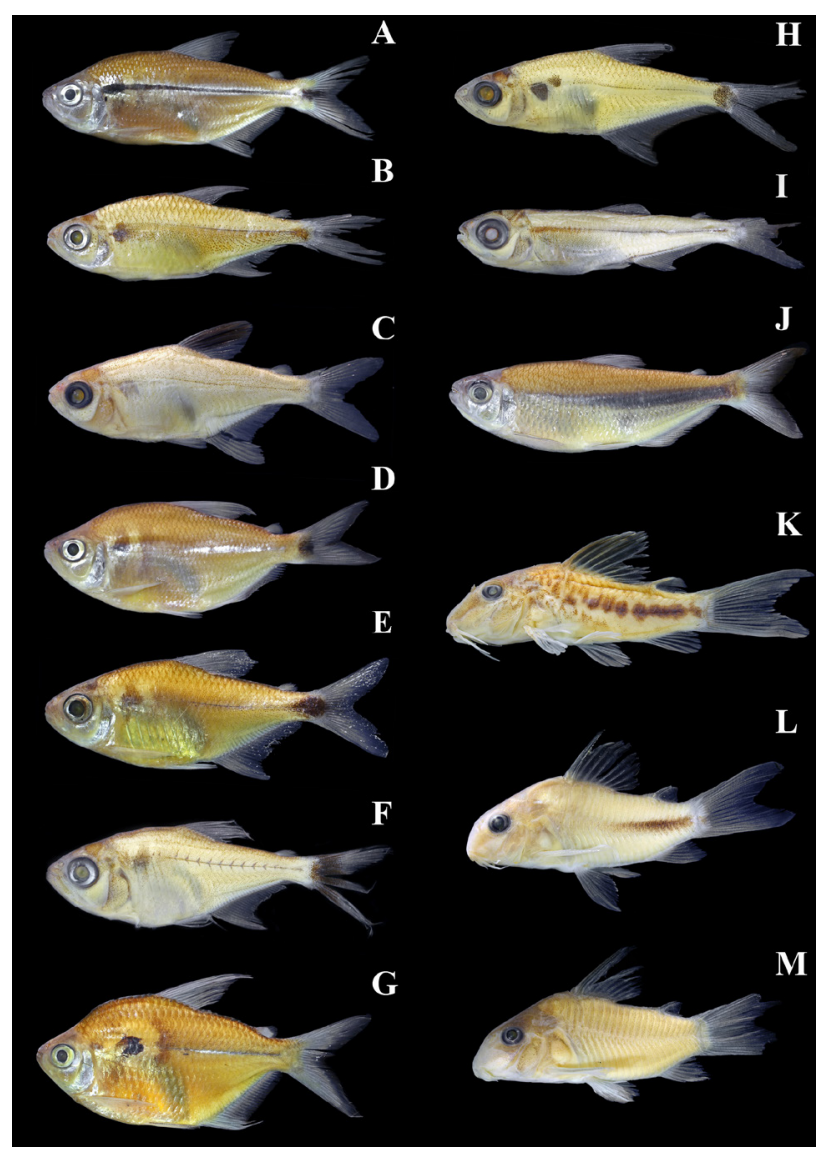

Figure 5. Examples of the fish species collected in the southern Guiana Shield tributaries of the Amazonas River, in the five state protected areas of the Northern Pará Drainage System, in northern Brazil. (A) Astyanax anterior, MPEG 18207, 81.6 mm SL. (B) Hyphessobrycon cf. agulha, MPEG 18181, 25.8 mm SL; (C) Hyphessobrycon georgettae, MPEG 34100, 19.0 mm SL; (D) Jupiaba abramoides, MPEG 18204, 70.8 mm SL; (E) Jupiaba atypindi, MPEG 17244, 39.8 mm SL; (F) Jupiaba potaroensis, MPEG 18159, 20.9 mm SL; (G) Moenkhausia chrysargyrea, MPEG 18204, 61.8 mm SL; (H) Phenacogaster simulata, MPEG 15597, 22.8 mm SL; (I) Bryconops aff. affinis, MPEG 18149, $22.3 \mathrm{~mm} \mathrm{SL;} \mathrm{(J)} \mathrm{Bryconops} \mathrm{cf.} \mathrm{colaroja,} \mathrm{MPEG}$ 17232, 94.5 mm SL; (K) Corydoras sp., MPEG 19162, 22.2 mm SL; (L) Corydoras baderi, MPEG 18238, 26.9 mm SL; (M) Corydoras guianensis, MPEG 15715, 25.8 mm SL. This figure is in color in the electronic version.

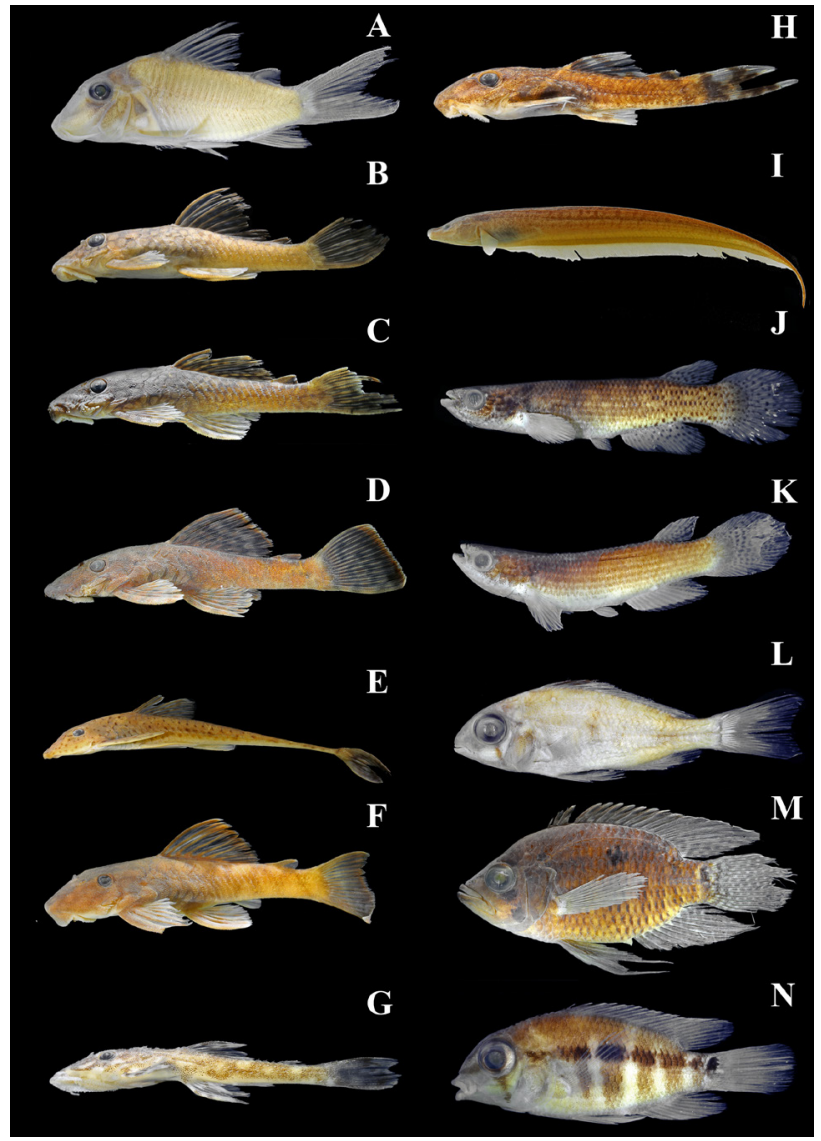

Figure 6. Examples of the fish species collected in the southern Guiana Shield tributaries of the Amazonas River, in the five state protected areas of the Northern Pará Drainage System, in northern Brazil. (A) Corydoras oxyrhynchus, MPEG 15716 , 27.4 mm SL. (B) Ancistrus sp.1, MPEG 17298, 57.2 mm SL; (C) Ancistrus sp.2, MPEG 17297, 58.7 mm SL; (D) Ancistrus sp.3, MPEG 19062, 48.0 mm SL; (E) Cteniloricaria napova, MPEG 34190, 121.9 mm SL; (F) Hemiancistrus sp., MPEG 19061, 70.3 mm SL; (G) Paralithoxus sp., MPEG 19135, 18.6 mm SL; (H) Peckoltia sp., MPEG 19137 14.9 mm SL; (I) Hypopomus artedi, MPEG 17301, 98.7 mm SL; (J) Anablepsoides sp.1, MPEG 17263, 54.6 mm SL; (K) Anablepsoides sp.3, MPEG 34703, 31.5 mm SL; (L) Acarichthys heckelii, MPEG 15485, 27.4 mm SL; (M) Acaronia nassa, MPEG 15398, $110.1 \mathrm{~mm} \mathrm{SL}$; (N) "Aequidens" potaroensis, MPEG 17302, $34.3 \mathrm{~mm}$ SL. This figure is in color in the electronic version.

\section{DISCUSSION}

In general, the composition of the fish fauna of the Northern Pará Drainage System was typical of the Neotropical region, with a predominance of otophysians (Characiformes, Siluriformes and Gymnotiformes) and cichlids (Roberts 1972; Lowe-McConnell 1999; Reis et al. 2016). While the data were not analyzed statistically, the clear variation in the fish diversity of the study areas may be accounted for, at least in part, by the positive relationship between fish diversity and the high productivity of the várzea floodplain ecosystems of the lowland Amazon basin (Forsberg et al. 1993), with decreasing diversity coinciding with the altitudinal gradient (Lujan $e t$ al. 2013). At one extreme, FLOTA Faro and FLOTA Paru, 
which are located in the lowest part of the study area, had the highest fish diversity, whereas the ESEC G-P North, located at the highest altitudes, had the smallest number of fish species. A decline in fish diversity at higher altitudes is typical of the Neotropical region (e.g., Albert et al. 2011; Lima and Ribeiro 2011; da Costa et al. 2018).

In addition to their greater productivity, Dagosta and de Pinna (2019) also discussed the role of the interconnectivity of lowland watercourses, which tend to facilitate the dispersal of taxa with more ample geographic ranges. By contrast, upland areas tend to be more isolated from neighboring watercourses, resulting in higher endemicity and reduced diversity in comparison with lowland areas. The altitudinal gradient may also represent a key environmental filter for the composition of the fish assemblage, reinforcing the complementarity of the features of different aquatic ecosystems in terms of the conservation of fish diversity. For example, loricariid species predominated in areas of higher altitude (FLOTA Trombetas and ESEC G-P North), while these fish were much less diverse in lowland areas. Areas of higher altitude typically have more rocky substrates and faster flowing water, with an abundance of microhabitats, a type of environment that favors some groups of loricariids (Teresa et al. 2016). In the lowland areas, the higher fish diversity recorded in our study may be related to the variety of aquatic ecosystems sampled, including lakes, streams, and large rivers, in contrast with the headwaters streams, that were the only bodies of water available for sampling in the upland areas.

Six species were recorded for the Amazon basin for the first time. Hyphessobrycon georgettae was previously known only from Suriname, while Jupiaba potaroensis and Phenacogaster simulata had only been recorded in Guyana, and J. keithi in French Guiana (Vari et al. 2009). Corydoras guianensis was known to occur in both French Guiana and Suriname, whereas C. oxyrhynchus was previously considered to be endemic to Suriname (Vari et al. 2009). These findings highlight the similarities of the fish faunas of the Northern Pará Drainage System (NPDS) and some of the coastal drainages of the Guiana Shield. This distribution pattern is described as the Guiana Shield (Atlantic and Amazonian versants) pattern, which may have been the result of faunal exchange caused by headwater capture events (Dagosta and de Pinna 2019). This pattern is further supported by the occurrence of the loricariid Parotocinclus halbothi and the heptapterid Pimelodella geryi Hoedeman 1961, which are known from both the NPDS and the Marowijne River in Suriname (Lehmann et al. 2014; Slobodian et al. 2017; Dagosta and de Pinna 2019). On the other hand, Cteniloricaria napova, Cyphocharax aninha, Hypomasticus lineomaculatus and Pimelodella humeralis (Covain et al. 2012; Birindelli et al. 2013; Wosiacki and Miranda 2013; Slobodian et al. 2017) appear to be endemic to the Northern Pará Drainage System.

\section{CONCLUSIONS}

The RAP assessment of the ichthyofauna of the Northern Pará Drainage System provided valuable insights into the fish diversity of the region, contributing an important database for future research and conservation programs. Further expeditions to the NPDS may nevertheless reduce gaps in the ichthyological, taxonomic, and geographic knowledge of this megadiverse group in the Amazon basin.

\section{ACKNOWLEDGMENTS}

We are grateful to the Secretaria Estadual de Meio Ambiente (SEMA), the Instituto do Homem e Meio Ambiente da Amazônia (IMAZON), and Conservaçấo Internacional do Brasil (CI-Brasil) for financial support. We would also like to thank the Rio Tinto Mining Company for logistic support. We are also grateful to Alexandre Aleixo, coordinator of the Project for the Diagnosis of the Biodiversity of the State Conservation Units of the Northern Pará Drainage System. We also thank A. Araujo, C. Ramos, D. Coutinho, J. Andrade, R. Raiol and W. Mota for participation in the fieldwork; A. Akama, A. Burbano, Â. Zanata, C. D. de Santana, D. Nielsen, F. Carvalho, F.C.T. Lima, H. Vera-Alcaraz, H. Varella, H. Gimenês, J. Birindelli, L. Sousa, M. Andrade, P. Ito, R. Reis, and W. Crampton for their help with the identification of species, I. Magalhães for curatorial assistance, and Murilo Pastana and Stephen Ferrari for the review of the language. The authors are supported by Fundação de Amparo à Pesquisa do Estado de São Paulo (FAPESP, grants 2018/09445-9 to GMD and 2018/05084-1 to LAWP), Coordenação de Aperfeiçoamento de Pessoal de Nível Superior (CAPES), and Conselho Nacional de Desenvolvimento Científico e Tecnológico (CNPq, grants 300940/2015-7 and 302497/2018-8 to WBW). This contribution was also supported by the Diversity and Evolution of Gymnotiformes Project (FAPESP/Smithsonian proc. 2016/19075-9).

\section{REFERENCES}

Abell, R.; Thieme, M.L.; Revenga, C.; Bryer, M.; Kottelat, M.; Bogutskaya, N.; et al. 2008. Freshwater ecoregions of the world: a new map of biogeographic units for freshwater biodiversity conservation. Bioscience, 58: 403-414.

Albert, J.S.; Petry, P.; Reis, R.E. 2011. Major biogeographic and phylogenetic patterns. In: Albert, J.S.; Reis, R.E. (Ed.). Historical Biogeography of Neotropical Freshwater Fishes. University of California Press, Berkeley. p.21-57.

Bernard, E. 2008. Inventários Biológicos Rápidos no Parque Nacional Montanhas do Tumucumaque, Amapá, Brasil. RAP Bulletin of Biological Assessment 48. Conservation International, Arlington, $145 \mathrm{p}$.

Birindelli, J.L.O.; Peixoto, L.A.W.; Wosiacki, W.B.; Britski, H.A. 2013. New species of Hypomasticus Borodin, 1929 (Characiformes: Anostomidae) from tributaries of the lower Rio Amazonas, Brazil. Copeia, 2013: 464-469. 
Brasil. 2000. Federal Lawei ${ }^{\circ} 9.985$, de 18 de julho de 2000. Federal legislation that institutes the Brazilian National System of Conservation Units. (http://www.planalto.gov.br/ccivil_03/ leis/L9985.htm). Accessed on 23 Dec 2019.

Brasil. 2014. Ministério do Meio Ambiente. Portaria no 445, de 17 dezembro de 2014. Official list of the Brazilian Ministry of Environment of species of fish and aquatic invertebrates threatened with extinction. (http://www.icmbio.gov.br/cepsul/ images/stories/legislacao/Portaria/2014/p_mma_445_2014_ lista_peixes_amea\%C3\%A7ados_extin\%C3\%A7\%C3\%A3o. pdf). Accessed on 01 Oct 2019.

da Costa, I.G.; Petry, A.C.; Mazzoni, M. 2018. Responses of fish assemblages to subtle elevations in headwater streams in southwestern Amazonia. Hydrobiologia, 809: 175-184.

Covain, R.; Fisch-Muller, S.; Montoya-Burgos, J.I.; Mol, J.H.; Le Bail, P.Y.; Dray, S. 2012. The Harttiini (Siluriformes: Loricariidae) from the Guianas: a multi-table approach to access their diversity, evolution, and distribution. Cybium, 36: 115-161.

Dagosta, F.C.P.; de Pinna, M. 2017. Biogeography of Amazonian fishes: deconstructing river basins as biogeographic units. Neotropical Ichthyology, 15: e170034.

Dagosta, F.C.P.; de Pinna, M. 2019. The fishes of the Amazon: distribution and biogeographical patterns, with a comprehensive list of species. Bulletin of the American Museum of Natural History, 2019: 1-163.

Fricke, R.; Eschmeyer, W.N.; Van der Laan, R (Eds.). 2019. Eschmeyer's catalog of fishes: genera, species, references. Institute for Biodiversity Science and Sustainability, San Francisco. (http:// researcharchive.calacademy.org/research/ichthyology/catalog/ fishcatmain.as). Accessed on 10 Dec 2019.

Ferreira, E.J.G. 1993. Composição, distribuição e aspectos ecológicos da ictiofauna de um trecho do rio Trombetas, na área de influência da futura UHE Cachoeira Porteira, estado do Pará, Brasil. Acta Amazonica, 23: 1-89.

Forsberg, B.R.; Araujo-Lima, C.A.R.M.; Martinelli, L.A. 1993. Autotrophic carbon sources for fish of the central amazon. Ecology, 74: 643-652.

IUCN. 2017. The IUCN Red List of Threatened Species. International Union for Conservation of Nature and Natural Resources. (http:// www.iucnredlist.org). Accessed on 02 Oct 2019.

Lehmann, P.; Lazzarotto, H.; Reis, R.E. 2014. Parotocinclus halbothi, a new species of small armored catfish (Loricariidae: Hypoptopomatinae), from the Trombetas and Marowijne River basins, in Brazil and Suriname. Neotropical Ichthyology, 12: 27-33.

Lima, F.C.T.; Ribeiro, A.C. 2011. Continental-scale tectonic controls of biogeography and ecology. In: Albert, J.S.; Reis, R.E. (Ed.). Historical Biogeography of Neotropical Freshwater Fishes. University of California Press, Berkeley. p.145-164.

Lowe-McConnell, R.H. 1999. Estudos Ecológicos em Comunidades de Peixes Tropicais. EDUSP, São Paulo, 534p.

Lujan, N.K.; Roach K.A; Jacobsen, D.; Winemiller, K.O.; Vargas, V.M.; Ching, V.R.; Maestre, J.A. 2013. Aquatic community structure across an Andes-to-Amazon fluvial gradient. Journal of Biogeography. 40: 1715-1728.
Nelson, J.S.; Grande, T.; Wilson, M.V.H. 2016. Fishes of the World, 5th ed. John Wiley and Sons, Hoboken, 752p.

Pará. 2007. Conselho Estadual de Meio Ambiente. Resolução no 54, 24 outubro 2007. Official list of fauna and flora threatened with extinction in the Brazilian state of Pará. (https://www. sema.pa.gov.br/2007/10/24/10059). Accessed on 02 Oct 2019.

Peel, M.C.; Finlayson, B.L.; McMahon, T.A. 2007. Updated world map of the Koppen-Geiger climate classification. Hydrology and Earth System Sciences Discussions, 4: 439-473.

Rapp Py-Daniel, L.H.; Deus, C.P.; Ribeiro, O.M.; L.M. Sousa. 2007. Peixes. In: Rapp Py-Daniel, L.H.; Deus, C.P.; Henriques, A. L.; Pimpão, D.M.; O.M. Ribeiro (Org). Biodiversidade do Médio Madeira: Bases Científicas para Propostas de Conservação. Editora INPA, Manaus. p.89-125.

Reis, R.E.; Albert, J.S.; Di Dario, F.; Mincarone, M.M.; Petry, P.; Rocha, L.A. 2016. Fish biodiversity and conservation in South America. Journal of Fish Biology, 89: 12-47.

Roberts, T.R. 1972. Ecology of fishes in the Amazon and Congo Basins. Bulletin of the Museum of Comparative Zoology, 143:117-147.

SEMA. 2010. Secretaria de Estado de Meio Ambiente do Pará. Plano de Manejo da Floresta Estadual do Paru. Imazon, Belém, 212p.

SEMA. 2011a. Secretaria de Estado de Meio Ambiente do Pará. Plano de Manejo da Estação Ecológica do Gräo-Pará, chapters I, II and III (cinco reinos, pesquisas e serviços ambientais). Imazon, Belém, 206p.

SEMA. 2011b. Secretaria de Estado de Meio Ambiente do Pará. Plano de Manejo da Floresta Estadual de Faro. Imazon, Belém, 178p.

SEMA. 2011c. Secretaria de Estado de Meio Ambiente do Pará. Plano de Manejo da Floresta Estadual do Trombetas. Imazon, Belém, 192p.

SEMA. 2011d. Secretaria de Estado de Meio Ambiente do Pará. Plano de manejo da Reserva Biológica Maicuru, chapters I, II and III (cinco reinos, pesquisas e serviços ambientais). Imazon, Belém, 164p.

Slobodian, V.; Akama, A.; Dutra, G.M. 2017. A new species of Pimelodella (Siluriformes: Heptapteridae) from the Guiana Shield, Brazil. Zootaxa, 4338: 85-100.

Teresa, F.B.; Souza, L.S.; Silva, D.M.A.; Barbosa, H.O.; Lima, J.D.; Nabout, J.C. 2016. Environmental constraints structuring fish assemblages in riffles: evidences from a tropical stream. Neotropical Ichthyology, 14: e150185.

Vari, R.P.; Ferraris, C.J.; Radosavljevic A.; Funk, V.A. 2009. Checklist of the freshwater fishes of the Guiana shield. Bulletin of the Biological Society of Washington, 17: 1-93.

Wosiacki, W.B.; Miranda, D.P.S. 2013. Description of a new small species of the genus Cyphocharax (Characiformes: Curimatidae) from the Lower Amazon basin. Copeia, 2013: 627-633.

RECEIVED: $20 / 05 / 2019$

ACCEPTED: 27/12/2019

ASSOCIATE EDITOR: Carlos David de Santana 


\section{SUPPLEMENTARY MATERIAL (only available in the electronic version)}

DUTRA et al. Rapid assessment of the ichthyofauna of the southern Guiana Shield tributaries of the Amazonas River in Pará, Brazil

Appendix S1. Metadata of sampling sites of RAP surveys (2008-2009) in five conservation units in the Northern Pará Drainage System in the southern Guiana Shield region of Pará state, Brazil. CS = collection site. FLOTA Faro = Faro State Forest, FLOTA Trombetas = Trombetas State Forest, FLOTA Paru = Paru State Forest, ESEC GrãoPará $=$ Grão-Pará Ecological Station, REBIO Maicuru = Maicuru Biological Reserve.

\begin{tabular}{|c|c|c|c|c|}
\hline Site & Conservation unit & Drainage & Habitat & Coordinates \\
\hline CS01 & FLOTA Faro & Rio Nhamundá & River channel & $1^{\circ} 42^{\prime} 50.3^{\prime \prime S} 57^{\circ} 14^{\prime} 49.1^{\prime \prime} \mathrm{W}$ \\
\hline $\mathrm{CSO} 2$ & FLOTA Faro & Rio Nhamundá & River channel & $1^{\circ} 43^{\prime} 49.2^{\prime \prime} \mathrm{S} 57^{\circ} 20^{\prime} 55.5^{\prime \prime} \mathrm{W}$ \\
\hline $\mathrm{CSO}$ & FLOTA Faro & Rio Nhamundá & Lake & $1^{\circ} 42^{\prime} 32.1^{\prime \prime S} 57^{\circ} 15^{\prime} 43.1^{\prime \prime} \mathrm{W}$ \\
\hline $\mathrm{CSO} 04$ & FLOTA Faro & Rio Nhamundá & Lake & $1^{\circ} 43^{\prime} 11.4^{\prime \prime} \mathrm{S} 57^{\circ} 13^{\prime} 32.5^{\prime \prime} \mathrm{W}$ \\
\hline CS05 & FLOTA Faro & Rio Nhamundá & Lake & $1^{\circ} 42^{\prime} 26^{\prime \prime S} 57^{\circ} 17^{\prime} 36.2^{\prime \prime} \mathrm{W}$ \\
\hline CSO6 & FLOTA Faro & Rio Nhamundá & Lake & $1^{\circ} 43^{\prime} 22.3^{\prime \prime S} 57^{\circ} 11^{\prime} 36.3^{\prime \prime} \mathrm{W}$ \\
\hline CSO7 & FLOTA Faro & Rio Nhamundá & River channel & $1^{\circ} 42^{\prime} 41.5^{\prime \prime S} 57^{\circ} 12^{\prime} 38.1^{\prime \prime} \mathrm{W}$ \\
\hline CS08 & FLOTA Faro & Rio Nhamundá & Stream & $1^{\circ} 50^{\prime} 43.5^{\prime \prime} \mathrm{S} 57^{\circ} 2^{\prime} 53.6^{\prime \prime} \mathrm{W}$ \\
\hline CSO9 & FLOTA Faro & Rio Nhamundá & Lake & $1^{\circ} 47^{\prime} 48^{\prime \prime S} 57^{\circ} 4^{\prime} 10^{\prime \prime} \mathrm{W}$ \\
\hline Porto Cikel & FLOTA Faro & Rio Nhamundá & River channel & $1^{\circ} 42^{\prime} 48.4^{\prime \prime} \mathrm{S} 57^{\circ} 12^{\prime} 48.4^{\prime \prime} \mathrm{W}$ \\
\hline Trail 1 & FLOTA Faro & Rio Nhamundá & Stream & $1^{\circ} 42^{\prime} 23.3^{\prime \prime S} 57^{\circ} 2^{\prime} 10.1^{\prime \prime} \mathrm{W}$ \\
\hline CS10 & FLOTA Trombetas & Rio Cuminapanema & River margin & $0^{\circ} 54^{\prime} \mathrm{S} 55^{\circ} 29^{\prime} \mathrm{W}$ \\
\hline CS11 & FLOTA Trombetas & Rio Cuminapanema & Stream & $0^{\circ} 58^{\prime} 18^{\prime \prime} \mathrm{S} 55^{\circ} 34^{\prime} \mathrm{W}$ \\
\hline $\mathrm{CS} 12$ & FLOTA Trombetas & Rio Cuminapanema & Stream & $0^{\circ} 57^{\prime} \mathrm{S} 55^{\circ} 35^{\prime} \mathrm{W}$ \\
\hline CS13 & FLOTA Trombetas & Rio Cuminapanema & Stream & $0^{\circ} 55^{\prime} 11.4^{\prime \prime} \mathrm{S} 55^{\circ} 29^{\prime} 42.4^{\prime \prime} \mathrm{W}$ \\
\hline CS14 & FLOTA Trombetas & Rio Cuminapanema & Stream & $0^{\circ} 56^{\prime} 11.2^{\prime \prime}$ S $55^{\circ} 30^{\prime} 3.17^{\prime \prime} \mathrm{W}$ \\
\hline CS15 & FLOTA Trombetas & Rio Cuminapanema & Stream & $0^{\circ} 56^{\prime} \mathrm{S} 55^{\circ} 29^{\prime} \mathrm{W}$ \\
\hline CS16 & FLOTA Trombetas & Rio Cuminapanema & Stream & $0^{\circ} 57^{\prime} \mathrm{S} 55^{\circ} 30^{\prime} \mathrm{W}$ \\
\hline CS17 & FLOTA Trombetas & Rio Cuminapanema & Stream & $0^{\circ} 57^{\prime} \mathrm{S} 55^{\circ} 30^{\prime} 38.9^{\prime \prime} \mathrm{W}$ \\
\hline CS18 & FLOTA Trombetas & Rio Cuminapanema & Stream & $0^{\circ} 57^{\prime} \mathrm{S} 55^{\circ} 32^{\prime} \mathrm{W}$ \\
\hline CS19 & FLOTA Trombetas & Rio Cuminapanema & Stream & $0^{\circ} 57^{\prime} 44.6^{\prime \prime} \mathrm{S} 55^{\circ} 32^{\prime} 13.4^{\prime \prime} \mathrm{W}$ \\
\hline CS20 & FLOTA Trombetas & Rio Cuminapanema & Stream & $0^{\circ} 57^{\prime} \mathrm{S} 55^{\circ} 31^{\prime} \mathrm{W}$ \\
\hline CS21 & FLOTA Trombetas & Rio Cuminapanema & Stream & $0^{\circ} 57^{\prime} \mathrm{S} 55^{\circ} 31^{\prime} \mathrm{W}$ \\
\hline CS22 & FLOTA Trombetas & Rio Cuminapanema & Stream & $0^{\circ} 57^{\prime} \mathrm{S} 55^{\circ} 31^{\prime} \mathrm{W}$ \\
\hline CS23 & FLOTA Trombetas & Rio Cuminapanema & Stream & $0^{\circ} 58^{\prime} \mathrm{S} 55^{\circ} 31^{\prime} 59^{\prime \prime} \mathrm{W}$ \\
\hline CS24 & ESEC South & Rio Curuá & Stream & $0^{\circ} 09^{\prime} 3.9^{\prime \prime S} 55^{\circ} 11^{\prime} 2.5^{\prime \prime} \mathrm{W}$ \\
\hline CS25 & ESEC South & Rio Curuá & Stream & $0^{\circ} 09^{\prime} 48.2^{\prime \prime} \mathrm{S} 55^{\circ} 11^{\prime} 5.2^{\prime \prime} \mathrm{W}$ \\
\hline CS26 & ESEC South & Rio Curuá & River channel & $0^{\circ} 08^{\prime} 58.7^{\prime \prime} \mathrm{S} 55^{\circ} 10^{\prime} 58.2^{\prime \prime} \mathrm{W}$ \\
\hline CS27 & ESEC South & Rio Curuá & Stream & $0^{\circ} 09^{\prime} 1.2^{\prime \prime} \mathrm{S} 55^{\circ} 11^{\prime} 3^{\prime \prime} \mathrm{W}$ \\
\hline CS28 & ESEC South & Rio Curuá & Stream & $0^{\circ} 11^{\prime} 10.7^{\prime \prime} 555^{\circ} 10^{\prime} 28.5^{\prime \prime} \mathrm{W}$ \\
\hline CS29 & ESEC South & Rio Curuá & Stream & $0^{\circ} 10^{\prime} 43.3^{\prime \prime} \mathrm{S} 55^{\circ} 10^{\prime} 38.9^{\prime \prime} \mathrm{W}$ \\
\hline CS30 & ESEC South & Rio Curuá & River channel & $0^{\circ} 09^{\prime} 52^{\prime \prime} \mathrm{S} 55^{\circ} 10^{\prime} 58.2^{\prime \prime} \mathrm{W}$ \\
\hline CS31 & ESEC South & Rio Curuá & Stream & $0^{\circ} 09^{\prime} 47.6^{\prime \prime S} 55^{\circ} 11^{\prime} 1.2^{\prime \prime} \mathrm{W}$ \\
\hline CS32 & ESEC South & Rio Curuá & River channel & $0^{\circ} 09^{\prime} 40.6^{\prime \prime} \mathrm{S} 55^{\circ} 10^{\prime} 27.8^{\prime \prime} \mathrm{W}$ \\
\hline CS33 & ESEC South & Rio Curuá & Stream & $0^{\circ} 09^{\prime} 40^{\prime \prime} \mathrm{S} 55^{\circ} 10^{\prime} 36.6^{\prime \prime} \mathrm{W}$ \\
\hline CS34 & ESEC South & Rio Curuá & Stream & $0^{\circ} 9^{\prime} 47.4^{\prime \prime} \mathrm{S} 55^{\circ} 11^{\prime} 22.7^{\prime \prime} \mathrm{W}$ \\
\hline CS35 & ESEC South & Rio Curuá & Lake & $0^{\circ} 09^{\prime} 47^{\prime \prime S} 55^{\circ} 11^{\prime} 20.9^{\prime \prime} \mathrm{W}$ \\
\hline CS36 & ESEC South & Rio Curuá & River channel & $0^{\circ} 09^{\prime} 21.3^{\prime \prime} 555^{\circ} 8^{\prime} 29^{\prime \prime} \mathrm{W}$ \\
\hline CS37 & ESEC South & Rio Curuá & Stream & $0^{\circ} 09^{\prime} 49.4^{\prime \prime} \mathrm{S} 55^{\circ} 11^{\prime} 52.9^{\prime \prime} \mathrm{W}$ \\
\hline CS39 & ESEC North & Rio Mapuera & Stream & $1^{\circ} 15^{\prime} 58.2^{\prime \prime} \mathrm{N} 58^{\circ} 41^{\prime} 4.3^{\prime \prime} \mathrm{W}$ \\
\hline CS40 & ESEC North & Rio Mapuera & Stream & $1^{\circ} 16^{\prime} 20.8^{\prime \prime} \mathrm{N} 58^{\circ} 41^{\prime} 9.2^{\prime \prime} \mathrm{W}$ \\
\hline CS41 & ESEC North & Rio Mapuera & Stream & $1^{\circ} 16^{\prime} 31.6^{\prime \prime} \mathrm{N} 58^{\circ} 41^{\prime} 11.5^{\prime \prime} \mathrm{W}$ \\
\hline CS42 & ESEC North & Rio Mapuera & Stream & $1^{\circ} 16^{\prime} 58.8^{\prime \prime} \mathrm{N} 58^{\circ} 42^{\prime} 9.1^{\prime \prime} \mathrm{W}$ \\
\hline CS43 & ESEC North & Rio Mapuera & Stream & $1^{\circ} 15^{\prime} 31.9^{\prime \prime} \mathrm{N} 58^{\circ} 42^{\prime} 40.8^{\prime \prime} \mathrm{W}$ \\
\hline CS44 & ESEC North & Rio Mapuera & Stream & $1^{\circ} 15^{\prime} 31.9^{\prime \prime} \mathrm{N} 58^{\circ} 42^{\prime} 40.8^{\prime \prime} \mathrm{W}$ \\
\hline CS45 & ESEC North & Rio Mapuera & Stream & $1^{\circ} 16^{\prime} 13.1^{\prime \prime} \mathrm{N} 58^{\circ} 42^{\prime} 16^{\prime \prime} \mathrm{W}$ \\
\hline CS46 & ESEC North & Rio Mapuera & Stream & $1^{\circ} 16^{\prime} 13.1^{\prime \prime} \mathrm{N} 58^{\circ} 42^{\prime} 16^{\prime \prime} \mathrm{W}$ \\
\hline CS47 & ESEC North & Rio Mapuera & Stream & $1^{\circ} 16^{\prime} 13.1^{\prime \prime} \mathrm{N} 58^{\circ} 42^{\prime} 16^{\prime \prime} \mathrm{W}$ \\
\hline CS50 & ESEC North & Rio Mapuera & Stream & $1^{\circ} 16^{\prime} 57.8^{\prime \prime} \mathrm{N} 58^{\circ} 41^{\prime} 19.3^{\prime \prime} \mathrm{W}$ \\
\hline CS51 & ESEC North & Rio Mapuera & Stream & $1^{\circ} 17^{\prime} 3.4^{\prime \prime} \mathrm{N} 58^{\circ} 41^{\prime} 20.4^{\prime \prime} \mathrm{W}$ \\
\hline CS52 & REBIO Maicuru & Rio Ipitinga & Stream & $0^{\circ} 51^{\prime} 13.8^{\prime \prime} \mathrm{S} 53^{\circ} 57^{\prime} 5.8^{\prime \prime} \mathrm{W}$ \\
\hline
\end{tabular}


Appendix S1. Continued.

\begin{tabular}{|c|c|c|c|c|}
\hline Site & Conservation unit & Drainage & Habitat & Coordinates \\
\hline $\mathrm{CS} 53$ & REBIO Maicuru & Rio Ipitinga & Stream & $0^{\circ} 49^{\prime} 59.2^{\prime \prime} \mathrm{S} 53^{\circ} 56^{\prime} 7.7^{\prime \prime} \mathrm{W}$ \\
\hline $\mathrm{CS} 54$ & REBIO Maicuru & Rio Ipitinga & Stream & $0^{\circ} 48^{\prime} 44.8^{\prime \prime} \mathrm{S} 53^{\circ} 56^{\prime} 55.7^{\prime \prime} \mathrm{W}$ \\
\hline CS55 & REBIO Maicuru & Rio Ipitinga & Stream & $0^{\circ} 49^{\prime \prime} 2^{\prime \prime} 553^{\circ} 56^{\prime} 27^{\prime \prime} \mathrm{W}$ \\
\hline CS56 & REBIO Maicuru & Rio Ipitinga & Stream & $0^{\circ} 49^{\prime} 51.9^{\prime \prime} \mathrm{S} 53^{\circ} 57^{\prime} 19.6^{\prime \prime} \mathrm{W}$ \\
\hline CS57 & REBIO Maicuru & Rio Ipitinga & Stream & $0^{\circ} 49^{\prime} 43.8^{\prime \prime} \mathrm{S} 53^{\circ} 56^{\prime} 53.4^{\prime \prime} \mathrm{W}$ \\
\hline CS58 & REBIO Maicuru & Rio Ipitinga & Stream & $0^{\circ} 48^{\prime} 33.7^{\prime \prime} \mathrm{S} 53^{\circ} 55^{\prime} 51.7^{\prime \prime} \mathrm{W}$ \\
\hline CS59 & REBIO Maicuru & Rio Ipitinga & River margin & $0^{\circ} 49^{\prime} 38.3^{\prime \prime} \mathrm{S} 53^{\circ} 55^{\prime} 29.6^{\prime \prime} \mathrm{W}$ \\
\hline CS60 & REBIO Maicuru & Rio Ipitinga & River margin & $0^{\circ} 49^{\prime} 0.4^{\prime \prime} \mathrm{S} 53^{\circ} 55^{\prime} 43.6^{\prime \prime} \mathrm{W}$ \\
\hline CS61 & REBIO Maicuru & Rio Ipitinga & Stream & $0^{\circ} 49^{\prime} 24.2^{\prime \prime} \mathrm{S} 53^{\circ} 55^{\prime} 46.4^{\prime \prime} \mathrm{W}$ \\
\hline CS62 & FLOTA Paru & Rio Paru & River channel & $0^{\circ} 55^{\prime} 54,7^{\prime \prime} 53^{\circ} 12^{\prime} 52,1^{\prime \prime} \mathrm{W}$ \\
\hline CS63 & FLOTA Paru & Rio Paru & River channel & $0^{\circ} 56^{\prime} 58.3^{\prime \prime} \mathrm{S} 53^{\circ} 14^{\prime} 31.4^{\prime \prime} \mathrm{W}$ \\
\hline CS64 & FLOTA Paru & Rio Paru & Stream & $0^{\circ} 59^{\prime} 21.6^{\prime \prime} 553^{\circ} 14^{\prime} 52.3^{\prime \prime} \mathrm{W}$ \\
\hline CS65 & FLOTA Paru & Rio Paru & Stream & $0^{\circ} 55^{\prime} 4.3^{\prime \prime} \mathrm{S} 53^{\circ} 15^{\prime} 17.6^{\prime \prime} \mathrm{W}$ \\
\hline CS67 & FLOTA Paru & Rio Paru & Stream & $0^{\circ} 55^{\prime} 4.5^{\prime \prime} \mathrm{S} 53^{\circ} 15^{\prime} 17.7^{\prime \prime} \mathrm{W}$ \\
\hline CS68 & FLOTA Paru & Rio Paru & Stream & $0^{\circ} 56^{\prime} 7.73^{\prime \prime S} 53^{\circ} 13^{\prime} 18.48^{\prime \prime} \mathrm{W}$ \\
\hline $\mathrm{CS} 69$ & FLOTA Paru & Rio Paru & Stream & $0^{\circ} 57^{\prime} 3.21^{\prime \prime} \mathrm{S} 53^{\circ} 15^{\prime} 37.8^{\prime \prime} \mathrm{W}$ \\
\hline CS70 & FLOTA Paru & Rio Paru & Stream & $0^{\circ} 55^{\prime} 54.7^{\prime \prime} 553^{\circ} 12^{\prime} 52.1^{\prime \prime} \mathrm{W}$ \\
\hline CS71 & FLOTA Paru & Rio Paru & Lake & $0^{\circ} 55^{\prime} 58.3^{\prime \prime} \mathrm{S} 53^{\circ} 12^{\prime} 53.8^{\prime \prime} \mathrm{W}$ \\
\hline CS72 & ESEC Centre & Rio Cuminapanema & Stream & $0^{\circ} 37^{\prime} 15.8^{\prime \prime} \mathrm{N} 55^{\circ} 43^{\prime} 31.9^{\prime \prime} \mathrm{W}$ \\
\hline CS73 & ESEC Centre & Rio Cuminapanema & Stream & $0^{\circ} 38^{\prime} 0^{\prime \prime} \mathrm{N} 55^{\circ} 42^{\prime} 50.5^{\prime \prime} \mathrm{W}$ \\
\hline CS74 & ESEC Centre & Rio Cuminapanema & Stream & $0^{\circ} 38^{\prime} 10.3^{\prime \prime} \mathrm{N} 55^{\circ} 43^{\prime} 24.1^{\prime \prime} \mathrm{W}$ \\
\hline CS75 & ESEC Centre & Rio Cuminapanema & Stream & $0^{\circ} 39^{\prime} 48.8^{\prime \prime} \mathrm{N} 55^{\circ} 43^{\prime} 51.9^{\prime \prime} \mathrm{W}$ \\
\hline CS76 & ESEC Centre & Rio Cuminapanema & Stream & $0^{\circ} 39^{\prime} 45.5^{\prime \prime} \mathrm{N} 55^{\circ} 43^{\prime} 5^{\prime} \mathrm{W}$ \\
\hline CS77 & ESEC Centre & Rio Cuminapanema & Stream & $0^{\circ} 39^{\prime} 8.9^{\prime \prime} \mathrm{N} 55^{\circ} 43^{\prime} 43.1^{\prime \prime} \mathrm{W}$ \\
\hline CS78 & ESEC Centre & Rio Cuminapanema & Stream & $0^{\circ} 38^{\prime} 22.3^{\prime \prime} \mathrm{N} 55^{\circ} 43^{\prime} 33.3^{\prime \prime} \mathrm{W}$ \\
\hline CS79 & ESEC Centre & Rio Cuminapanema & Stream & $0^{\circ} 38^{\prime} 15.3^{\prime \prime} \mathrm{N} 55^{\circ} 43^{\prime} 31.2^{\prime \prime} \mathrm{W}$ \\
\hline
\end{tabular}

Appendix S2. Voucher codes of fish collected during RAP surveys (2008-2009) in five conservation units in the Northern Pará Drainage System in the southern Guiana Shield region of Pará state, Brazil.

CLUPEIFORMES. Engraulidae. Amazonsprattus scintilla: MPEG 15488. Anchovia surinamensis: MPEG 14784. Anchoviella cayennensis: MPEG 15425. Anchoviella guianensis: MPEG 14882. Anchoviella lepidentostole: MPEG 15432. Pristigasteridae. Ilisha amazonica: MPEG 14776, MPEG 14847, MPEG 14893. Pellona castelnaeana: MPEG 14874. Pellona flavipinnis: MPEG 14896.

CHARACIFORMES. Acestrorhynchidae. Acestrorhynchus falcatus: MPEG 15761, MPEG 15787, MPEG 18187, MPEG 23359, MPEG 23366, MPEG 23367, MPEG 23370, MPEG 23378. Acestrorhynchus heterolepis: MPEG 23376. Acestrorhynchus microlepis: MPEG 14794, MPEG 14806, MPEG 14830, MPEG 14832, MPEG 14836, MPEG 14895, MPEG 14895. Acestrorhynchus nasutus: MPEG 15373, MPEG 15394. Gnathocharax steindachneri: MPEG 15349, MPEG 15396, MPEG 15420. Heterocharax macrolepis: MPEG 15120, MPEG 15379. Anostomidae. Anostomoides laticeps: MPEG 14787, MPEG 14867. Anostomus ternetzi: MPEG 15694, MPEG 15725. Hypomasticus lineomaculatus: MPEG 15573, MPEG 15589, MPEG 15663, MPEG 15697, MPEG 15729. Laemolyta proxima: MPEG 14766, MPEG 14771. Laemolyta taeniata: MPEG 14772. Leporinus agassizii: MPEG 15400. Leporinus amazonicus: MPEG 14812. Leporinus brunneus: MPEG 15402, MPEG 15407. Leporinus cf. cylindriformis: MPEG 24317, MPEG 24318. Leporinus fasciatus: MPEG 14767, MPEG 24320, MPEG 24322, MPEG 33991. Leporinus friderici: MPEG 33992, MPEG 33993, MPEG 33994. Leporinus cf. granti: MPEG 33995. Leporinus jatuncochi: MPEG 24319. Leporinus cf. megalepis: MPEG 24326. Leporinus melanostictus: MPEG 33996. Leporinus aff. nijsseni: MPEG 33997, MPEG 33998, MPEG 33999, MPEG 34000. Leporinus tigrinus: MPEG 24321. Leporinus sp.1: MPEG 34005. Leporinus sp.2: MPEG 34001, MPEG 34003, MPEG 34004. Bryconidae. Brycon pesu: MPEG 14770, MPEG 23306, MPEG 23321, MPEG 23334, MPEG 34006, MPEG 34007, MPEG 34008. Chalceidae. Chalceus macrolepidotus: MPEG 23307, MPEG 23309, MPEG 23317, MPEG 23325. Characidae. Acestrocephalus sardina : MPEG 14773. Aphyocharax sp.: MPEG 15411. Astyanax anterior: MPEG 18207, MPEG 18208, MPEG 18209. Astyanax bimaculatus: MPEG 15655, MPEG 15808, MPEG 34115, MPEG 34116, MPEG 34117, MPEG 34118, MPEG 34119, MPEG 34120, MPEG 34121, MPEG 34122, MPEG 34123, MPEG 34124, MPEG 34125, MPEG 34126, MPEG 34127, MPEG 34128, MPEG 34129, MPEG 34130 , MPEG 34131, MPEG 34132, MPEG 34133, MPEG 34134, MPEG 34135, MPEG 34136, MPEG 34137, MPEG 34138, MPEG 34139, MPEG 34140, MPEG 34141. Astyanax cf. multidens: MPEG 15382. Brachychalcinus orbicularis: MPEG 
23316, MPEG 23338. Brittanichthys myersi: MPEG 15111, MPEG 15419. Bryconamericus orinocoensis: MPEG 15354, MPEG 15387, MPEG 15427. Charax caudimaculatus: MPEG 34143, MPEG 34144, MPEG 34145. Hemibrycon surinamensis: MPEG 34082. Hemigrammus analis: MPEG 15107, MPEG 15384, MPEG 15435. Hemigrammus bellottii: MPEG 15338, MPEG 15351, MPEG 15399, MPEG 34740, MPEG. 34733. Hemigrammus coeruleus: MPEG 15323, MPEG 34146. Hemigrammus geisleri: MPEG 15742. Hemigrammus hyanuary: MPEG 15412. Hemigrammus lunatus: MPEG 15563, MPEG 15577, MPEG 15594, MPEG 15602, MPEG 15632, MPEG 15657, MPEG 15658, MPEG 15666, MPEG 15678, MPEG 15788, MPEG 15789, MPEG 18160, MPEG 18167. Hemigrammus microstomus: MPEG 15423, MPEG 34734. Hemigrammus ocellifer: MPEG 15327, MPEG 15344, MPEG 15375, MPEG 15408, MPEG 34097, MPEG 34098, MPEG 34099, MPEG 34722. Hemigrammus schmardae: MPEG 15118, MPEG 15331. Hemigrammus stictus: MPEG 15433. Hemigrammus sp.1: MPEG 34083, MPEG 34084, MPEG 34147, MPEG 34148, MPEG 34710, MPEG 34711, MPEG 34714, MPEG 34715, MPEG 34718, MPEG 34719, MPEG 34725. Hemigrammus sp.2: MPEG 15593, MPEG 15656, MPEG 18150, MPEG 18155, MPEG 18164, MPEG 18169, MPEG 18175, MPEG 18180, MPEG 18196, MPEG 18201, MPEG 34149, MPEG 34150. Hyphessobrycon cf. agulha: MPEG 18154, MPEG 18170, MPEG 18179, MPEG 18181, MPEG 18195, MPEG 18198, MPEG 18200. Hyphessobrycon diancistrus: MPEG 15380, MPEG 15418. Hyphessobrycon cf. minor: MPEG 34151. Hyphessobrycon georgettae: MPEG 18197, MPEG 34100, MPEG 34101, MPEG 34102, MPEG 34103, MPEG 34104, MPEG 34105, MPEG 34106, MPEG 34107, MPEG 34108, MPEG 34712. Hyphessobrycon socolof: MPEG 15377, MPEG 15421. Jupiaba abramoides: MPEG 16637, MPEG 18206. Jupiaba acanthogaster: MPEG 15705, MPEG 15736. Jupiaba atypindi: MPEG 16639, MPEG 16640, MPEG 17226, MPEG 17227, MPEG 17229, MPEG 17230, MPEG 17243, MPEG 17244, MPEG 17245, MPEG 17246, MPEG 17247, MPEG 17248, MPEG 17250, MPEG 34085. Jupiaba keithi: MPEG 15572, MPEG 15667, MPEG 15776, MPEG 15793. Jupiaba meunieri: MPEG 18186. Jupiaba polylepis: MPEG 15585, MPEG 15689, MPEG 15723, MPEG 15755, MPEG 15777, MPEG 15796, MPEG 15797, MPEG 23341. Jupiaba potaroensis: MPEG 18148, MPEG 18153, MPEG 18159, MPEG 18163, MPEG 18194. Knodus cf. heteresthes: MPEG 15707. Knodus hypopterus: MPEG 34086, MPEG 34087, MPEG 34088, MPEG 34089, MPEG 34090, MPEG 34091, MPEG 34092, MPEG 34093. Knodus aff. hypopterus: MPEG 15617, MPEG 15634. Knodus sp.1: MPEG 34094, MPEG 34095. Knodus sp.2: MPEG 34096. Microschemobrycon callops: MPEG 15711, MPEG 15752. Microschemobrycon casiquiare: MPEG 34109. Moenkhausia celibela: MPEG 15710, MPEG 34735. Moenkhausia chrysargyrea: MPEG 18204. Moenkhausia collettii: MPEG 15385, MPEG 15426, MPEG 15580, MPEG 15739, MPEG 34160. Moenkhausia cotinho: MPEG 15430. Moenkhausia megalops: MPEG 34110 , MPEG 34111, MPEG 34112, MPEG 34113. Moenkhausia mikia: MPEG 15576, MPEG 15579, MPEG 15621, MPEG 15633, MPEG 15677, MPEG 15770, MPEG 15786, MPEG 34161. Moenkhausia oligolepis: MPEG 15574, MPEG 15645, MPEG 15760, MPEG 15769, MPEG 15802, MPEG 15807, MPEG 15811, MPEG 18151, MPEG 18156, MPEG 18157, MPEG 18158, MPEG 18172, MPEG 18173, MPEG 18176, MPEG 18178, MPEG 18185, MPEG 23268, MPEG 23270, MPEG 23273, MPEG 23274, MPEG 23275, MPEG 23276, MPEG 23279, MPEG 23281, MPEG 23286, MPEG 23287, MPEG 23289, MPEG 23290, MPEG 23292, MPEG 23293, MPEG 23295, MPEG 23300, MPEG 23337, MPEG 23342. Moenkhausia aff. surinamensis: MPEG 18161, MPEG 34152, MPEG 34153, MPEG 34154, MPEG 34155, MPEG 34156, MPEG 34157, MPEG 34158, MPEG 34159, MPEG 34707, MPEG 34723, MPEG 34724. Phenacogaster simulata: MPEG 15571, MPEG 15597, MPEG 15603, MPEG 15615, MPEG 15628, MPEG 15636, MPEG 15660, MPEG 15669, MPEG 15688, MPEG 15704, MPEG 15753, MPEG 34172, MPEG 34721. Poptella brevispina: MPEG 17225, MPEG 17228, MPEG 17233, MPEG 17234, MPEG 17235, MPEG 17236, MPEG 17237, MPEG 17238, MPEG 17239, MPEG 17240, MPEG 17242, MPEG 17251, MPEG 18166, MPEG 18177, MPEG 18203, MPEG 15738, MPEG 34162, MPEG 34163 , MPEG 34164, MPEG 34165, MPEG 34166, MPEG 34200, MPEG 34201. Rhinopetitia sp.: MPEG 15709, MPEG 15741, MPEG 34736. Roeboexodon guyanensis: MPEG 15708, MPEG 15740. Serrapinnus gracilis: MPEG 15564, MPEG 15598, MPEG 15599, MPEG 15737, MPEG 15772, MPEG 15790, MPEG 15791, MPEG 15795, MPEG 34737, MPEG 34114, MPEG 34167, MPEG 34168, MPEG 34169, MPEG 34170, MPEG 34171, MPEG 34708, MPEG 34709, MPEG 34713 , MPEG 34716, MPEG 34717, MPEG 34720. Tetragonopterus carvalhoi: MPEG 15722, MPEG 15751. Tetragonopterus chalceus: MPEG 23310, MPEG 23312, MPEG 23315, MPEG 23319, MPEG 23330, MPEG 23332. Thayeria sp.: MPEG 15340. Chilodontidae. Caenotropus labyrinthicus: MPEG 23320, MPEG 23331, MPEG 23333. Crenuchidae. Characidium cf. etheostoma: MPEG 18097, MPEG 24299, MPEG 24300, MPEG 24302, MPEG 24303, MPEG 24304, MPEG Characidium zebra: MPEG 15560, MPEG 15590, MPEG 15601, MPEG 15610, MPEG 15620, MPEG 15630, MPEG 15643, MPEG 15654, MPEG 15664, MPEG 15674, MPEG 15698, MPEG 15730, MPEG 15765, MPEG 15781, MPEG 18098, MPEG 18099, MPEG 18100, MPEG 18101, MPEG 18216, MPEG 24301. Crenuchus spilurus: MPEG 15339. Microcharacidium eleotrioides: MPEG 15561, MPEG 15592, MPEG 15611, MPEG 15612, MPEG 15644, MPEG 15675. Ctenoluciidae. Boulengerella cuvieri: MPEG 33935, MPEG 33936, MPEG 33937, MPEG 33938, MPEG 33939, MPEG 33940, MPEG 33941, MPEG 33942. Boulengerella lucius: MPEG 14817, MPEG 14866, MPEG 33943, MPEG 33944, MPEG 33945 , 
MPEG. Boulengerella maculata: MPEG 14765, MPEG 14769, MPEG 14779, MPEG 14780, MPEG 14795, MPEG 14835, MPEG 14892. Boulengerella xyrekes: MPEG 14786, MPEG 14868, MPEG 14878. Curimatidae. Curimata knerii: MPEG 34009, MPEG 34010. Curimata ocellata: MPEG 15106, MPEG 34180. Curimatopsis macrolepis: MPEG 15383. Curimatopsis melanura: MPEG 15335, MPEG 35300. Cyphocharax abramoides: MPEG 14883. Cyphocharax aninha: MPEG 15591, MPEG 15767, MPEG 15783, MPEG 15810, MPEG 34174, MPEG 34175. Cyphocharax festivus: MPEG 34011. Cyphocharax helleri: MPEG 15734. Cyphocharax nigripinnis: MPEG 15334, MPEG 15416. Cyphocharax cf. notatus: MPEG 15104, MPEG 34012. Cyphocharax spiluropsis: MPEG 15701, MPEG 15732, MPEG 15782, MPEG 34013, MPEG 34014, MPEG 34015, MPEG 34016, MPEG 34017, MPEG 34018, MPEG 34019, MPEG 34020, MPEG 34021. Cyphocharax spilurus: MPEG 15328, MPEG 15372, MPEG 15699, MPEG 15731, MPEG 34022. Steindachnerina amazonica: MPEG 15700, MPEG 15733. Cynodontidae. Cynodon septenarius: MPEG 14793, MPEG 14809, MPEG 14828, MPEG 14850, MPEG 14886. Hydrolycus armatus: MPEG 23363. Hydrolycus tatanaia: MPEG 23362, MPEG 23364, MPEG 23374. Erythrinidae. Erythrinus erythrinus: MPEG 15357, MPEG 15401, MPEG 17276, MPEG 17278, MPEG 17281, MPEG 17282, MPEG 17283, MPEG 17284, MPEG 18137, MPEG 18138, MPEG 18140, MPEG 18144, MPEG 18199, MPEG 23260, MPEG 23261, MPEG 23262, MPEG 23263, MPEG 23264, MPEG 23265, MPEG 23269, MPEG 23277, MPEG 23280, MPEG 23288, MPEG 23291, MPEG 23339. Hoplerythrinus unitaeniatus: MPEG 15361, MPEG 15798, MPEG 17279, MPEG 17304, MPEG 23267, MPEG 23271, MPEG 23282, MPEG 23283, MPEG 23284, MPEG 23298, MPEG 23311. Hoplias aimara: MPEG 15727, MPEG 15728 Hoplias curupira: MPEG 15358, MPEG 15362, MPEG 15696, MPEG 15780, MPEG 15799, MPEG 18141, MPEG 18143, MPEG 23266, MPEG 23278, MPEG 23285, MPEG 23297, MPEG 23301, MPEG 23343, MPEG Hoplias malabaricus: 15366, MPEG MPEG 15588, MPEG 15610, MPEG 15757, MPEG 15758, MPEG 15764, MPEG 15800, MPEG 15801, MPEG 23272, MPEG 23294, MPEG 23299, MPEG 23302, MPEG 23340. Gasteropelecidae. Carnegiella marthae: MPEG 15348, MPEG 15397. Carnegiella strigata: MPEG 15342. Gasteropelecus sternicla: MPEG 15559, MPEG 15587, MPEG 15662, MPEG 15693, MPEG 15724, MPEG 15763, MPEG 15779. Hemiodontidae. Argonectes longiceps: MPEG 14796, MPEG 14827. Bivibranchia fowleri: MPEG 14774. Hemiodus argenteus: MPEG 14879, MPEG 34029, MPEG 34030. Hemiodus immaculatus: MPEG 14785, MPEG 14818, MPEG 14837, MPEG 14853, MPEG 14870, MPEG 14877, MPEG 14971, MPEG 14972, MPEG 15105. Hemiodus iratapuru: MPEG 34023, MPEG 34024, MPEG 34025, MPEG 34026, MPEG 34027, MPEG 34028. Hemiodus semitaeniatus: MPEG 14778, MPEG 14802, MPEG 14804, MPEG 14894. Hemiodus unimaculatus: MPEG 34031, MPEG 34032, MPEG 34033, MPEG 34034, MPEG 34035, MPEG 34036, MPEG 34037. Micromischodus sugillatus: MPEG 14782. Iguanodectidae. Bryconops aff. affinis: MPEG 18149, MPEG 18171. Bryconops alburnoides: MPEG 14790, MPEG 14824, MPEG 14839, MPEG 14856, MPEG 14860, MPEG 34738. Bryconops aff. caudomaculatus: MPEG 15703. Bryconops aff. colanegra: MPEG 15613, MPEG 15614, MPEG 18162, MPEG 18165, MPEG 18183, MPEG 18184, MPEG 18202. Bryconops cf. colaroja: MPEG 17232. Bryconops cf. collettei: MPEG 34188. Bryconops disruptus: MPEG 15112. Bryconops giacopinii: MPEG 34038. Bryconops aff. melanurus: MPEG 34187. Bryconops sp.: MPEG 34189. Iguanodectes cf. geisleri: MPEG 15325. Iguanodectes cf. spilurus: MPEG 15415. Lebiasinidae. Copella arnoldi: MPEG 15557, MPEG 15558, MPEG 15586, MPEG 15600, MPEG 15619, MPEG 15640, MPEG 15651, MPEG 15652, MPEG 15673, MPEG 15778, MPEG Copella nattereri: MPEG 15108, MPEG 15117, MPEG 15329, MPEG 15429. Nannostomus digrammus: MPEG 15115, MPEG 15381, MPEG 15431. Nannostomus eques: MPEG 15371. Nannostomus marginatus: MPEG 15333, MPEG 15346, MPEG 15417. Nannostomus trifasciatus: MPEG 15413. Nannostomus unifasciatus: MPEG 15110, MPEG 15370, MPEG 15374. Pyrrhulina cf. maxima: MPEG 14873, MPEG 15629, MPEG 15661, MPEG 15672, MPEG 15692, MPEG 18110, MPEG 18111, MPEG 18112, MPEG 18113, MPEG 18114, MPEG 18115, MPEG 18116, MPEG 18117, MPEG 18188, MPEG 18214, MPEG 19110, MPEG 19111, MPEG 19112, MPEG 19113, MPEG 19114, MPEG 19115, MPEG 19116, MPEG 19117, MPEG 19118, MPEG 19119, MPEG 19120, MPEG 19121, MPEG 19122, MPEG 19123, MPEG 19124. Pyrrhulina sp.: MPEG 15330, MPEG 15345. Parodontidae. Parodon guyanensis: MPEG 23258, MPEG 23259. Prochilodontidae. Prochilodus rubrotaeniatus: MPEG 15631, MPEG 15759, MPEG 34039, MPEG 34040, MPEG 34041, MPEG 34042. Serrasalmidae. Mylesinus paraschomburgkii: MPEG 23313. Myloplus arnoldi: MPEG 23335. Myloplus rhomboidalis: MPEG 23303, MPEG 23322, MPEG 23324, MPEG 23326. Myloplus rubripinnis: MPEG 23305. Piaractus brachypomus: MPEG 23308. Pygopristis denticulata: MPEG 15422. Serrasalmus altispinis: MPEG 14861, MPEG 14865, MPEG 14890, MPEG 23304, MPEG 23323. Serrasalmus eigenmanni: MPEG 23314, MPEG 23318, MPEG 23328, MPEG 23336. Serrasalmus hastatus: MPEG 23327. Serrasalmus manueli: MPEG 14799, MPEG 14801, MPEG 14803, MPEG 14852, MPEG 14862, MPEG 14884, MPEG 15405. Serrasalmus rhombeus: MPEG 23329. Serrasalmus sp.: MPEG 14808. Triportheidae. Agoniates halecinus: MPEG 14764, MPEG 14797, MPEG 14816, MPEG 14831, MPEG 14854, MPEG 14859, MPEG 14900. Triportheus albus: MPEG 14788, MPEG 14789, MPEG 14811, MPEG 14834, MPEG 14843, MPEG 14849, MPEG 14869, MPEG 14881, MPEG 34196, MPEG 34197, MPEG 34198, MPEG 34199. 
SILURIFORMES. Auchenipteridae. Ageneiosus inermis: MPEG 22792. Ageneiosus polystictus: MPEG 14819, MPEG 14840, MPEG 14891, MPEG 14898. Ageneiosus lineatus: MPEG 14805, MPEG 14822, MPEG 14857. Auchenipterichthys longimanus: MPEG 14800, MPEG 14810, MPEG 14821, MPEG 14826, MPEG 14844, MPEG 14846, MPEG 14863, MPEG 15355. Auchenipterus brachyurus: MPEG 14815, MPEG 14833, MPEG 34078, MPEG 34079, MPEG 34080. Auchenipterus nuchalis: MPEG 14777, MPEG 14871, MPEG 14880. Centromochlus heckelii: MPEG 14838, MPEG 14858. Tatia intermedia: MPEG 23355, MPEG 23356, MPEG 23358. Tatia nigra: MPEG 14783, MPEG 14820, MPEG 14842, MPEG 14851, MPEG 14887. Tatia orca: MPEG 14823. Trachelyichthys decaradiatus: MPEG 15109. Trachelyopterichthys taeniatus: MPEG 14876. Trachelyopterus galeatus: MPEG 24331, MPEG 24332, MPEG 24333, MPEG 24334, MPEG 24335, MPEG 24336. Callichthyidae. Callichthys callichthys: MPEG 15756, MPEG 17295, MPEG 19163, MPEG 19165, MPEG 19168, MPEG 19170, MPEG 19173, MPEG 19175, MPEG 19177, MPEG 19178, MPEG 19179, MPEG 19180, MPEG 19181, MPEG 34081. Corydoras baderi: MPEG 18238. Corydoras guianensis: MPEG 15565, MPEG 15581, MPEG 15715, MPEG 15716, MPEG 15746. Corydoras oxyrhynchus: MPEG 15747. Corydoras sp.: MPEG 19162. Hoplosternum littorale: MPEG 15356, MPEG 15359, MPEG 15367. Megalechis picta: MPEG 15343 15360, MPEG 15363. Cetopsidae. Helogenes marmoratus: MPEG 14876, MPEG 15605, MPEG 15622, MPEG 18104, MPEG 18105, MPEG 18106, MPEG 18107, MPEG 18108, MPEG 18109, MPEG 18147, MPEG 18218, MPEG 18219, MPEG 23344, MPEG 23345, MPEG 23346, MPEG 23347, MPEG 23348, MPEG 23349, MPEG 23350, MPEG 23351, MPEG 23352, MPEG 23353, MPEG 23354 , MPEG 23357. Doradidae. Acanthodoras cataphractus: MPEG 15365. Acanthodoras spinosissimus: MPEG 15116. Anduzedoras oxyrhynchus: MPEG 14841. Nemadoras elongatus: MPEG 14889. Oxidoras niger: MPEG 22793. Scorpiodoras heckelii: MPEG 14845, MPEG 15369. Tenellus leporhinus: MPEG 14848, MPEG 14864, MPEG 14888. Trachydoras brevis: MPEG 14813. Heptapteridae. Gladioglanis conquistador: MPEG 15347. Imparfinis hasemani: MPEG 22789, MPEG 22790, MPEG 22791. Pariolius armillatus: MPEG 22784. Pimelodella cristata: MPEG 34142, MPEG 34177. Pimelodella geryi: MPEG 15690, MPEG 15691. Pimelodella humeralis: MPEG 15712, MPEG 15744, MPEG 34994. Pimelodella leptosoma: MPEG 15743. Rhamdia quelen: MPEG 18213, MPEG 34176, MPEG 34178, MPEG 34179, MPEG 34181, MPEG 34182, MPEG 34183, MPEG 34184, MPEG 34185, MPEG 34186. Loricariidae. Ancistrus sp.1: MPEG 17298. Ancistrus sp.2: MPEG 17296, MPEG 17297. Ancistrus sp.3: MPEG 19062. Ancistrus sp.4: MPEG 19068. Baryancistrus hadrostomus: MPEG 19064, MPEG 19065, MPEG 19066. Cteniloricaria napova: MPEG 34190. Hemiancistrus sp.: MPEG 19061. Curculionichthys sp.: MPEG 15607, MPEG 15714, MPEG 15745. Hypostomus carinatus: MPEG 15103. Hypostomus hemicochliodon: MPEG 22794, MPEG 22796, MPEG 22797, MPEG 34191. Hypostomus sp.: MPEG 22795, MPEG 34192, MPEG 34193. Otocinclus vittatus: MPEG 19063, MPEG 19125, MPEG 19176, MPEG 34194. Paralithoxus sp.: MPEG 19135. Parotocinclus halbothi: MPEG 17299. Peckoltia sp.: MPEG 19136, MPEG 19137, MPEG 19138. Pseudancistrus sp.1: MPEG 19067. Pseudancistrus sp.2: MPEG 34195. Rineloricaria stewarti: MPEG 19140. Rineloricaria sp.: MPEG 15713, MPEG 18123, MPEG 18124, MPEG 18240, MPEG 19141, MPEG 19142, MPEG 19144, MPEG 19145, MPEG 19146, MPEG 19147, MPEG 19148, MPEG 19149, MPEG 19150, MPEG 19151, MPEG 19152, MPEG 19153, MPEG 19154, MPEG 19155, MPEG 19156, MPEG 19157, MPEG 19158. Pimelodidae. Hemisorubim platyrhynchos: MPEG 23361, MPEG 23371, MPEG 23375. Hypophthalmus fimbriatus: MPEG 14829. Hypophthalmus marginatus: MPEG 14798. Pimelodus albofasciatus: MPEG 14763, MPEG 14775, MPEG 14781, MPEG 14814, MPEG 14899. Pimelodus ornatus: MPEG 23368, MPEG 23372, MPEG 23373. Pinirampus pirinampu: MPEG 23360, MPEG 23369. Pseudoplatystoma punctifer: MPEG 23377. Sorubim elongatus: MPEG 14825 , MPEG 14855, MPEG 14902. Pseudopimelodidae. Batrochoglanis villosus: MPEG 23365. Microglanis poecilus: MPEG 15487. Trichomycteridae. Ituglanis amazonicus: MPEG 15556, MPEG 15618, MPEG 15639, MPEG 15780, MPEG 17285, MPEG 17286, MPEG 17287, MPEG 18118, MPEG 18119, MPEG 18220, MPEG 19083, MPEG 19084, MPEG 19085, MPEG 19086, MPEG 19087, MPEG 19088, MPEG 19089, MPEG 19090, MPEG 19091. Paracanthopoma parva: MPEG 15486. Stegophilus panzeri: MPEG 15718. Stenolicmus ix: MPEG 15101.

GYMNOTIFORMES. Gymnotidae. Electrophorus voltai: MPEG 15529. Gymnotus carapo: MPEG 15680, MPEG 18235, MPEG 22787. Gymnotus coropinae: MPEG 15566, MPEG 15608, MPEG 15609, MPEG 15624, MPEG 15625, MPEG 15647, MPEG 15648, MPEG 15681, MPEG 15682, MPEG 15683, MPEG 15773, MPEG 15774, MPEG 18211, MPEG 18233, MPEG 18234, MPEG 18236, MPEG 18237. Gymnotus cf. stenoleucus: MPEG 18134, MPEG 19130, MPEG 22788. Gymnotus sp.: MPEG 18135, MPEG 19128, MPEG 19129, MPEG 19131. Hypopomidae. Brachyhypopomus beebei: MPEG 15646, MPEG 15336, MPEG 15679. Brachyhypopomus pinnicaudatus: MPEG 19126. Brachyhypopomus sullivani: MPEG 15623. 18125, MPEG 18126, MPEG 18127, MPEG 18133, MPEG 18230, MPEG 18231, MPEG 18232. Hypopomus artedi: MPEG 17301. Rhamphichthyidae. Hypopygus lepturus: MPEG 15119, MPEG 15332, MPEG 15392.

CYPRINODONTIFORMES. Rivulidae. Anablepsoides sp.1: MPEG 17253, MPEG 17255, MPEG 17256, MPEG 17257, MPEG 17258, MPEG 17259, MPEG 17260, MPEG 17261, MPEG 17262, MPEG 17263, MPEG 17264, MPEG 17265, 
MPEG 17266, MPEG 17267, MPEG 17269, MPEG 17270, MPEG 17271, MPEG 17272, MPEG 17273, MPEG 17274, MPEG 17275, MPEG 17305. Anablepsoides sp.2: MPEG 34704, MPEG 34705. Anablepsoides sp.3: MPEG 34698, MPEG 34699, MPEG 34700, MPEG 34701, MPEG 34702, MPEG 34703. Laemosemion sp.1: MPEG 15805. Laemosemion sp.2: MPEG 15642, MPEG 15653. Laemosemion sp.3: MPEG 15341, MPEG 15424.

GOBIIFORMES. Eleotridae. Microphilypnus ternetzi: MPEG 15326.

SYNBRANCHIFORMES. Synbranchidae. Synbranchus marmoratus: MPEG 15671, MPEG 18102, MPEG 18103, MPEG 22781, MPEG 22783, MPEG 22785, MPEG 22786.

CICHLIFORMES. Cichlidae. Acarichthys heckelii: MPEG 15485. Acaronia nassa: MPEG 15398. "Aequidens" potaroensis: MPEG 17288, MPEG 17289, MPEG 17290, MPEG 17291, MPEG 17292, MPEG 17293, MPEG 17302, MPEG 17303. Aequidens tetramerus: MPEG 15350, MPEG 15364, MPEG 15368. Aequidens sp.1: MPEG 15762, MPEG 15794, MPEG 15804, MPEG 15803, MPEG 18225, MPEG 33954, MPEG 33955. Aequidens sp.2: MPEG 33949, MPEG 33950, MPEG 33951, MPEG 33952, MPEG 33953, MPEG 34706. Aequidens sp.3.: MPEG 18128, MPEG 18129, MPEG 18130, MPEG 18131, MPEG 18132, MPEG 18189, MPEG 18190, MPEG 18191, MPEG 18192, MPEG 18193, MPEG 18221, MPEG 18222, MPEG 18223, MPEG 18224, MPEG 18226, MPEG 18227, MPEG 33946, MPEG 33947. Apistogramma agassizii: MPEG 15494, MPEG 15495. Apistogramma cf. pertensis: MPEG 15491. Apistogramma gr. regani: MPEG 15492. Apistogramma sp.: MPEG 22798. Caquetaia spectabilis: MPEG 15496, MPEG 15497, MPEG 33956. Cichla jariina: MPEG 34731. Crenicichla albopunctata: MPEG 15567, MPEG 15568, MPEG 15650, MPEG 15685, MPEG 15719, MPEG 15748. Crenicichla cf. inpa: MPEG 15569, MPEG 15582, MPEG 15583, MPEG 18120, MPEG 18229, MPEG 18121, MPEG 18122, MPEG 24305, MPEG 24306, MPEG 24307, MPEG 24308, MPEG 24309, MPEG 24310. Crenicichla menezesi: MPEG 18228, MPEG 24311, MPEG 24312. Crenicichla pydanielae: MPEG 24313. Crenicichla regani: MPEG 15489. Crenicichla saxatilis: MPEG 15595, MPEG 17294, MPEG 24314, MPEG 34739. Crenicichla strigata: MPEG 24315. Geophagus altifrons: MPEG 34726, MPEG 34727, MPEG 34730. Krobia guianensis: MPEG 15570, MPEG 15584, MPEG 15596, MPEG 15626, MPEG 15627, MPEG 15635, MPEG 15668, MPEG 15687, MPEG 15720, MPEG 15721, MPEG 15749, MPEG 15750, MPEG 15775. Mesonauta acora: MPEG 15388. Mesonauta festivus: MPEG 15395, MPEG 34729. Satanoperca acuticeps: MPEG 15406. Satanoperca jurupari: MPEG 24328, MPEG 24329, MPEG 24330. Taeniacara candidi: MPEG 15490, MPEG 15493.

PERCIFORMES. Sciaenidae. Plagioscion squamosissimus: MPEG 14875. 